\title{
Cathepsin D deficiency delays central nervous system myelination by inhibiting proteolipid protein trafficking from late endosome/lysosome to plasma membrane
}

\author{
Da-Zhi Guo ${ }^{1,2,6}$, Lin Xiao ${ }^{3,6}$, Yi-Jun Liu ${ }^{4}$, Chen Shen ${ }^{5}$, Hui-Fang Lou ${ }^{4}$, Yan $\mathrm{Lv}^{1}$ and Shu-Yi Pan ${ }^{1}$
}

This study aimed to investigate the role of cathepsin D (CathD) in central nervous system (CNS) myelination and its possible mechanism. By using CathD knockout mice in conjunction with immunohistochemistry, immunocytochemistry and western blot assays, the myelination of the CNS and the development of oligodendrocyte lineage cells in vivo and in vitro were observed. Endocytosis assays, real-time-lapse experiments and total internal reflection fluorescence microscopy were used to demonstrate the location and movement of proteolipid protein in oligodendrocyte lineage cells. In addition, the relevant molecular mechanism was explored by immunoprecipitation. The increase in Fluoromyelin Green staining and proteolipid protein expression was not significant in the corpus callosum of CathD ${ }^{-1-}$ mice at the age of P11, P14 and P24. Proteolipid protein expression was weak at each time point and was mostly accumulated around the nucleus. The number of oligodendrocyte lineage cells (olig2+) and mature oligodendrocytes (CC1+) significantly decreased between P14 and P24. In the oligodendrocyte precursor cell culture of CathD $\mathrm{D}^{-/-}$mice, the morphology of myelin basic protein-positive mature oligodendrocytes was simple while oligodendrocyte precursor cells showed delayed differentiation into mature oligodendrocytes. Moreover, more proteolipid protein gathered in late endosomes/lysosomes (LEs/Ls) and fewer reached the plasma membrane. Immunohistochemistry and immunoelectron microscopy analysis showed that CathD, proteolipid protein and VAMP7 could bind with each other, whereas VAMP7 and proteolipid protein colocalized with CathD in late endosome/lysosome. The findings of this paper suggest that CathD may have an important role in the myelination of CNS, presumably by altering the trafficking of proteolipid protein.

Experimental \& Molecular Medicine (2018) 50, e457; doi:10.1038/emm.2017.291; published online 16 March 2018

\section{INTRODUCTION}

CathD (Cathepsin D) is a lysosomal, aspartic endoproteinase that requires an acidic $\mathrm{pH}$ to be proteolytically active. Although the level of its expression in different cells varies considerably, CathD is expressed in all tissues ${ }^{1}$ and has been involved in many fundamental functions of cells, such as the degradation of intracellular proteins in the lysosomal compartment, apoptosis, inflammation and tumor progression. ${ }^{2-4}$ Mutations in the CathD gene cause fatal neurological diseases, which are characterized by an extensive loss of neurons and myelin, pronounced gliosis and the accumulation of lipofuscin within the remaining cells in human infants, as well as some lysosomal storage disorders. ${ }^{5,6} \mathrm{CathD}^{-/-}$mice, generated by gene targeting, also develop neurological diseases resembling those in human, characterized by signs including the accumulation of storage materials in neurons as well as neurodegeneration, which is particularly significant within the thalamus, the hippocampus and the deep laminae of the cerebral cortex. ${ }^{7,8}$ Several reports also revealed the disruption of myelin sheaths within the corpus callosum and the thalamus in CathD-/mice. ${ }^{9,10}$ Recent in vivo neurobiochemistry studies indicated that myelin-related proteolipid protein (PLP) and myelin basic protein (MBP) were both markedly reduced at P24, and myelin sheaths became significantly atrophic in CathD-/- mice. ${ }^{11}$ Furthermore, there was a pronounced accumulation of cholesteryl esters and abnormal levels of proteins related to cholesterol transport. ${ }^{11}$ However, the mechanism underlying the defects in myelin sheaths of CathD - / - mice remains unknown.

\footnotetext{
${ }^{1}$ Department of Hyperbaric Oxygen, Navy General Hospital of PLA, Beijing, China; ${ }^{2}$ Cerebrovascular Disease Center of ChangHai Hospital, Second Military Medical University, Shanghai, China; ${ }^{3}$ Institute of Neuroscience and Key Laboratory of Molecular Neurobiology of Ministry of Education, Shanghai, China; ${ }^{4}$ Institute of Neuroscience, University of Zhejiang, Hangzhou, China and ${ }^{5}$ Company's Office of Service Center, China Petroleum and Natural Gas Group Corporation, Beijing, China

6These authors contributed equally to this work.

Correspondence: Professor S-Y Pan, Department of Hyperbaric Oxygen, Navy General Hospital of PLA, No. 6, Fucheng Road, Haidian District, Beijing 100048, China.

E-mail: syp201002@sina.com

Received 12 July 2017; revised 16 August 2017; accepted 28 August 2017
} 
As the most abundant protein present in the central nervous system (CNS) myelin of higher vertebrates, PLP ( $\sim 80 \%$ of the total myelin proteins) has a low molecular weight and is a highly hydrophobic proteolipid protein containing four transmembrane domains that interact with the cholesterol and galactosylceramide-enriched membranes during its biosynthetic transport in oligodendrocytes. ${ }^{12-14}$ Different from other CNS myelin proteins such as MBP, myelin-associated glycoprotein (MAG) and CNP, PLP is synthesized in membrane-bound polysomes, followed by the incorporation in the endoplasmic reticulum (ER) membrane, further processing through the Golgi apparatus, and vesicular transport to the myelin membrane. ${ }^{15}$ When initially expressed in cultured oligodendrocytes, PLP resides in a compartment showing the characteristics of late endosome/lysosome (LEs/L). ${ }^{16}$ Co-culture with neurons leads to an increased level of PLP on the plasma membrane and its detachment from the LEs/L, followed by the maturation of oligodendrocytes. Such observation has supported the idea that the amount of PLP in LEs/Ls and on the plasma membrane of oligodendrocytes is regulated by neural signal molecules. ${ }^{6}$ Recently, Anke Feldmann et al. ${ }^{17}$ demonstrated that PLP is transported to the cell surface from LEs/Ls via distinct trafficking pathways. Hence, we postulate that CathD deficiency may affect the metabolism or the transport of PLP in oligodendrocyte lineage cells (OLs).

In this study, the development of OLs and their intracellular PLP transportation were investigated using CathD knockout mice. It was found that myelin maturation and OL development were delayed by inhibiting the movement of PLP from LEs/Ls to the plasma membrane of the OLs. The findings shown in this paper may point to a new potential mechanism underlying the myelin defects caused by CathD deficiency.

\section{MATERIALS AND METHODS}

\section{Experimental animals}

Heterozygous mice (C57/BL CathD ${ }^{+/-}$) carrying a mutation in the CathD protein, obtained from Christoph Peters (Institute of Molecular Medicine and Cell Research, University of Freiburg, Freiburg, Germany), were used to generate homozygous animals for the experiment. The use and care of animals followed the guidelines of the Zhejiang University Animal Research Advisory Committee. Using the polymerase chain reaction and oligonucleotide primers as described previously, ${ }^{8}$ the genotype of individual animals was determined from the genomic DNA isolated from a small sample of the tail.

\section{Immunoelectron microscopy}

For immunoelectron microscopy, mice were deeply anesthetized and perfused with $1 \%$ paraformaldehyde, $0.2 \%$ picric acid and $0.05 \%$ glutaraldehyde in a $0.1 \mathrm{~m}$ phosphate buffer ( $\mathrm{pH}$ 7.4). Ultrathin sections of $80 \mathrm{~nm}$ thickness processed from Lowicryl-embedded blocks of the brain were placed on coated nickel grids and incubated for $45 \mathrm{~min}$ in a blocking solution consisting of $10 \%$ human serum albumin in $0.05 \mathrm{M}$ Tris-buffered saline and $0.03 \%$ Triton X-100 (TBST). The grids were incubated with PLP antibodies (1:1000\#x0FF1B;polyclone; Abcam, Shanghai, China) and CathD antibodies (1:200; N-19; polyclone; SantaCruz, Shanghai, China) in TBST with $2 \%$ human serum albumin (HSA) at $28^{\circ} \mathrm{C}$ overnight. After rinsing, the grids were incubated for
$3 \mathrm{~h}$ with donkey anti-rabbit IgG antibodies conjugated to colloidal gold particles ( $5 \mathrm{~nm}$, Sigma-Aldrich Corp., St Louis, MO, USA) and donkey anti-goat IgG antibodies conjugated to colloidal gold particles ( $15 \mathrm{~nm}$, Sigma) diluted 1:30 in 2\% HSA and $0.5 \%$ polyethylene glycol in TBST. The grids were then soaked in Tris-buffered saline for $30 \mathrm{~min}$ and counterstained for electron microscopy, using saturated aqueous uranyl acetate followed by lead citrate.

\section{Western blot analysis}

CathD $^{+/+}$and CathD ${ }^{-/-}$mice at the age of P11, P14 and P24 were deeply anesthetized using $10 \%$ chloral hydrate and subsequently perfused transcardially with ice-cold phosphate-buffered saline (PBS). The brain was carefully isolated and subsequently homogenized in a radioimmunoprecipitation assay buffer (Beyotime, Shanghai, China), using a hand-held homogenizer. Protein concentration was determined using a Bio-Rad protein assay. Thirty milligrams of each sample were separated on a 10 or $12 \%$ Tris $\mathrm{HCl}$ gel (Bio-Rad Laboratories, Hercules, CA, USA) and transferred to a polyvinylidene fluoride (PVDF) membrane. Membranes were blocked in $0.1 \%$ Tween 20 TBS containing $5 \%$ nonfat dry milk for $1 \mathrm{~h}$ before incubation with primary antibodies. Primary antibodies were diluted in the same solution as follows: anti-PLP (1:1000; Abcam), anti-CNP (1:500; Sigma), anti-Olig2 (1:500; Millipore, Burlington, MA, USA), anti- $\beta$ actin (1:15 000; Sigma), anti-CathD (1:800, N-19; SantaCruz) and anti-VAMP11 (1:500; Abcam). Samples were incubated with antimouse, anti-rabbit or anti-goat secondary antibodies conjugated to horseradish peroxidase for $1 \mathrm{~h}$ before the proteins were detected using an Immun-StarTM Western CTM detection system (Bio-Rad Laboratories), the blots were exposed using ChemiDocTM XRS+ with Image labTM (Bio-Rad Laboratories) and band intensities were measured using Image-lab software (Bio-Rad Laboratories). Protein expression was normalized to $\beta$-actin.

\section{Immunohistochemistry and immunocytochemistry}

For immunohistochemistry, the brain was isolated and fixed with icecold $4 \%$ paraformaldehyde in PBS overnight at $4{ }^{\circ} \mathrm{C}$ and subsequently cryoprotected using $30 \%$ sucrose in PBS. The brain was embedded in optimal cutting temperature compound (OCT) (Tissue-Tek, Sakura, Torrance, CA, USA) and sliced into 10 or $20 \mu \mathrm{m}$ sections using a freezing microtome. Tissue sections underwent immunostaining, first by blocking and permeabilizing in $10 \%$ bovine serum albumin $/ 0.3 \%$ Triton X-100, followed by overnight incubation at $4{ }^{\circ} \mathrm{C}$ with corresponding primary antibodies. For immunocytochemistry, cultured cells were fixed by $4 \%$ paraformaldehyde in PBS at $4{ }^{\circ} \mathrm{C}$ for $10 \mathrm{~min}$ and were permeabilized with $0.2 \%$ Triton X-100 in PBS for $10 \mathrm{~min}$, or with methanol for $10 \mathrm{~min}$ at $-20^{\circ} \mathrm{C}$. Dilutions for primary antibodies were as follows: rabbit anti-PLP (1:1000; Abcam), rabbit anti-NG2 (1:200; Chemicon, Rolling Meadows, IL, USA), mouse antiAPC/CC1 (1:500; Abcam), rat anti-MBP (1:200; Chemicon), rabbit anti-cleaved caspase-3 (1:200; Cell Signaling Technology, Danvers, MA, USA), rabbit anti-Olig2 (1:200; Millipore), mouse anti-LAMP1 (1:200; Assay designs, Hines Drive, Ann Arbor, MI, USA), anti-NG2 (1:300; Millipore), rat anti-MBP (1:200; Chemicon), rabbit anti-PLP (1:1000; Abcam), mouse anti-O10 (1:50; R\&D, Minneapolis, MN, USA), goat anti-CathD ${ }^{-/-}$(1:200; SantaCruz), mouse anti-LAMP1 (1:200; Assay designs) and mouse anti-VAMP11 (1:200; Abcam). Samples were incubated with corresponding primary antibodies overnight at $4{ }^{\circ} \mathrm{C}$, followed by rinsing in PBS and staining with Cy3 (1:1000; Invitrogen, Carlsbad, CA, USA) or Alexa Flour 488conjugated secondary antibodies (1:1000; Invitrogen) for $1 \mathrm{~h}$ at room 
temperature. After antibody incubation, some brain sections were stained with FluoroMyelin Green Fluorescent Myelin Stain (1:300; Invitrogen) for $20 \mathrm{~min}$ at room temperature. For samples with a determined number of total cells, their nuclei were counterstained for 5 min with $100 \mathrm{~nm}$ 4, 6-diamidino-2-phenylindole dihydrochloride (Invitrogen).

\section{Isolation and culture of mouse oligodendrocyte precursor cells}

The following procedures were modified based on the Chen et al. ${ }^{18}$ In a laminar flow hood, the embryos of CathD ${ }^{+/+}$and CathD ${ }^{-/-}$mice were removed on the embryonic day (E)14.5-17.5 and placed in clean Petri dishes containing ice-cold HBSS. The embryos were decapitated and their skins on the telencephalic bulb and the skull were removed gently from the head by holding the neck region with a pair of forceps. The telencephalic bulb was detached using a pair of 451 angled Dumont forceps. The medial portion was removed while the lateral part of the cerebral cortex was retained. The cortex was placed in a clean Petri dish containing ice-cold HBSS and the meninges were removed using the pair of forceps. Each cortex was cut into two to three pieces, which were transferred into an ice-cold neurosphere growth medium $(0.5 \mathrm{ml}$ per brain) containing $20 \mathrm{ng}$ ml-1 epidermal growth factor (EGF) and $20 \mathrm{ng} \mathrm{ml}^{-1}$ basic fibroblast growth factor (bFGF). The cortices were dissociated by mechanical trituration using a fire-polished glass Pasteur pipette ( $\sim 35$ strokes) until the cell suspension had no or very few small clumps. The suspension was kept on ice for $2 \mathrm{~min}$ and subsequently passed through a $50-\mathrm{mm}$ nylon pouch placed on a $15-\mathrm{ml}$ conical tube to obtain a single cell suspension. The cells were counted with a hemocytometer and added into a six-well plate $(4 \mathrm{ml}$ of neurosphere growth medium per well) at a concentration of $5 \times 10^{4}$ cells per $\mathrm{ml}$. The plate was incubated in an incubator at $37^{\circ} \mathrm{C}$ and $5 \% \mathrm{CO}_{2}$. The cells were fed once every 2 days by replacing half of the medium with a fresh neurosphere growth medium. Near the fourth day after the formation of neurospheres, the EGF/bFGF-containing neurosphere growth medium was gradually changed to a B104 CM-containing oligosphere medium by replacing one-fourth of the former medium with the latter medium every other day in a period of 2 weeks. The oligospheres should be either mechanically dissociated using a fire-polished pipette as described above, followed by passage through a $50-\mathrm{mm}$ nylon mesh to obtain single cells, or by using an enzymatic treatment of trypsin. The single cells were resuspended in $4 \mathrm{ml}$ oligosphere medium, spun down at $120 \mathrm{~g}$ (1000 r.p.m.) for $5 \mathrm{~min}$ at RT and passed through a 50-mm nylon mesh to obtain a cell suspension. The cell suspension was cultured in the oligosphere medium on an uncoated plate at a density of $\sim 3 \times 10^{4}$ cells per ml. Oligospheres would be formed again after 5-7 days. Alternatively, the cell suspension could be plated on a poly-ornithine-coated plate in an oligodendrocyte precursor cell (OPC) medium to achieve OPC proliferation, or be cultured in an oligodendrocyte differentiation medium to achieve differentiation.

\section{Endocytosis assay}

Endocytosis was assessed by antibody internalization as described previously. ${ }^{17}$ OLs from CathD ${ }^{+/+}$and $\mathrm{CathD}^{-/-}$mice were incubated on ice for $45 \mathrm{~min}$ with mouse anti-O10 monoclonal antibodies bond to PLP extracellular epitopes in DMEM containing 10\% horse serum, followed by incubation with goat anti-mouse $\mathrm{Cy} 3$ antibodies (Red) for $30 \mathrm{~min}$ on ice. The cells were incubated at $37^{\circ} \mathrm{C}$ to allow endocytosis and were subsequently incubated with anti-goat Alexa Flour 488 (Green) to stain the proteins on the cell surface. Finally, the cells were fixed in PBS containing 4\% paraformaldehyde. In the assay, the signal of endocytosed epitopes would appear solely in the red channel, whereas the signal of surface-localizing epitopes would appear in both red and green channels. The percentage of endocytosing cells was determined from three independent experiments (100 cells were counted in each experiment).

\section{Microscopy}

Fluorescence images were acquired on a Confocal microscope (Fluoview 1000; Olympus; or Fluoview 500; Olympus, Tokyo, Japan) equipped with a ULPLANAPO $\times 60 / 1.2 \mathrm{w}$ immersion objective (Olympus). The images were taken with a resolution of $1024 \times 1024$. The gain of the photomultiplier was adjusted to maximize the signal/ noise ratio without causing image saturation.

For live cell imaging, coverslips containing the cells were mounted in a live cell imaging chamber and observed in ECS $(\mathrm{NaCl} 145 \mathrm{~mm}$, $\mathrm{KCl} 3 \mathrm{~mm}, \mathrm{CaCl}_{2} 3 \mathrm{~mm}, \mathrm{MgCl}_{2} \bullet 6 \mathrm{H}_{2} \mathrm{O} 2 \mathrm{~mm}$, HEPES $10 \mathrm{~mm}$, Glucose $8 \mathrm{~mm}, \mathrm{pH}$ 7.4) at room temperature. Time-lapse imaging was performed on a confocal laser scanning microscope (IX71; Olympus). Images were acquired at free intervals for the indicated time, using sequential line excitations at 488 and $543 \mathrm{~nm}$ and appropriate band pass emission filters. Image processing and analysis were performed using Image-Pro Plus5.1 (Media Cybernetics, Shanghai, China). Quantification of colocalization was performed using the colocalization module of the software. Vesicle movement was analyzed by subtracting from each image the information in its preceding image of a time stack. The different image stack generated from the above operation was used to identify vesicle motility events. TIRFM (total internal reflection fluorescence microscopy) was performed on an Olympus IX81 inverted microscope equipped with a $\times 601.45$ NA oil immersion objective (PlanApoN, Olympus). Evanescent field excitation was obtained by focusing 488- and 568-nm laser lights that resulted in a field depth of $\sim 100 \mathrm{~nm}$. Images were acquired with a cooled CCD camera (iXon, ANDOR Technology, Beijing, China) controlled by Andor iQ software (Version2.0, Media Cybernetics, Shanghai, China). Each pixel corresponded to $133 \mathrm{~nm}$ in the specimen plane.

\section{Biotinylation}

Biotinylation of cell surface proteins was carried out using a modified approach of Duan et al. ${ }^{19}$ After $1 \mathrm{~h}$ of preincubation and $6 \mathrm{~min}$ of HBSS wash, the cells were rinsed twice with $\mathrm{HBSS} / \mathrm{Ca}^{2+}-\mathrm{Mg}^{2+}$ containing $138 \mathrm{NaCl}, 2.7 \mathrm{KCl}, 1.5 \mathrm{KH}_{2} \mathrm{PO}_{4}, 9.6 \mathrm{Na}_{2} \mathrm{HPO}_{4}, 1 \mathrm{MgCl}_{2}$ and $0.1 \mathrm{CaCl}_{2}$ (mM, pH 7.3). The cells were then incubated in sulfoNHS-biotin solution $\left(1 \mathrm{mg} \mathrm{ml}{ }^{-1}\right.$ in $\left.\mathrm{HPBS} / \mathrm{Ca}^{2+}-\mathrm{Mg}^{2+}\right)$ for $20 \mathrm{~min}$ at $4^{\circ} \mathrm{C}$. Biotinylation was terminated by washing twice in a quenching solution of $\mathrm{HBSS} / \mathrm{Ca}^{2+}-\mathrm{Mg}^{2+}$, in which the $\mathrm{NaCl}$ content was replaced by an equimolar concentration of $100 \mathrm{~mm}$ glycine (to maintain the $300 \mathrm{mOsm}$ ). This was followed by an additional $45 \mathrm{~min}$ incubation in the quenching solution at $4{ }^{\circ} \mathrm{C}$. Subsequently, the quenching solution was removed and the cells were lysed with $100 \mathrm{ml}$ per well of a radioimmunoprecipitation assay buffer containing protease inhibitors (100 mm Tris-HCl, pH 7.4, $150 \mathrm{~mm} \mathrm{NaCl,} 1$ mm EDTA, 1\% Triton $\mathrm{X}-100,1 \%$ sodium deoxycholate, $0.1 \%$ SDS, $1 \mathrm{~mm}$ iodoacetamide, $1 \mathrm{mg} \mathrm{ml}^{-1}$ leupeptin, $5 \mathrm{mg} \mathrm{ml}^{-1}$ aprotinin and $250 \mathrm{~mm}$ phenylmethylsulfonyl fluoride). The lysis step was continued for $1 \mathrm{~h}$ at $4{ }^{\circ} \mathrm{C}$ with vigorous shaking. The lysates were centrifuged at $14000 \mathrm{~g}$ for $15 \mathrm{~min}$ at $4{ }^{\circ} \mathrm{C}$. An aliquot of $300 \mathrm{ml}$ of the supernatant was used as the whole-cell fraction for western blot analysis. The remaining supernatant $(600 \mathrm{ml})$ was incubated with $300 \mathrm{ml}$ of avidin bead 
suspension for $1 \mathrm{~h}$ at room temperature with gentle shaking. The avidin-lysate solution was then centrifuged for $15 \mathrm{~min}$ at $14000 \mathrm{~g}$, and the supernatant was collected as the intracellular fraction for western blot analysis. The pellet was washed four times with $1 \mathrm{ml}$ radioimmunoprecipitation assay buffer and resuspended in $300 \mathrm{ml}$ Laemmli buffer (62.4 mM Tris-HCl, pH 7, 2\% SDS, 20\% glycerol and 5\% 2-mercaptethanol) for $1 \mathrm{~h}$ with gentle shaking at room temperature. After centrifugation for $15 \mathrm{~min}$ at $14000 \mathrm{~g}$, the supernatant was collected as the biotinylated (plasma membrane) fraction for western blot analysis.

\section{Immunoprecipitation}

Briefly, the tissue samples were harvested as described above and homogenated in $1 \mathrm{ml}$ of lysis buffer (1\% Triton X-100, $50 \mathrm{~mm}$ Tris, $\mathrm{pH}$ 7.4, $150 \mathrm{~mm} \mathrm{NaCl}, 1 \mu \mathrm{g} \mathrm{ml}{ }^{-1}$ aprotinin, $5 \mu \mathrm{g} \mathrm{ml} \mathrm{m}^{-1}$ leupeptin, $1 \mu \mathrm{g} \mathrm{ml}^{-1}$ pepstatin, $1 \mathrm{~mm}$ phenylmethanesulfonyl fluoride (PMSF) and $1 \mathrm{~mm} \mathrm{~N}$-ethylmaleimide). After the nuclei were removed (5 min, 3200 r.p.m.) and nonspecific binding to goat IgG was eliminated, the lysates were incubated with polyclonal goat anti-CathD and goat antiPLP antibodies overnight at $4{ }^{\circ} \mathrm{C}$, followed by protein A/G-Sepharose for $1 \mathrm{~h}$ at $4{ }^{\circ} \mathrm{C}$ to precipitate the IgG. The pelleted beads were washed five times in $50 \mathrm{~mm}$ Tris- $\mathrm{HCl}, \mathrm{pH} 7.2,150 \mathrm{~mm} \mathrm{NaCl}, 1 \%$ Triton $\mathrm{X}-100,1 \%$ deoxycholate, $0.1 \%$ SDS, $1 \mathrm{~mm}$ 1,4-dithiothreitol, $1 \mathrm{~mm} \mathrm{~N}-$ ethylmaleimide, $1 \mu \mathrm{g} \mathrm{ml}^{-1}$ aprotinin, $5 \mu \mathrm{g} \mathrm{ml}^{-1}$ leupeptin, $1 \mu \mathrm{g} \mathrm{ml}{ }^{-1}$ pepstatin and $1 \mathrm{~mm}$ PMSF. Finally, the beads were resuspended in a SDS-PAGE sample buffer and heated for $5 \mathrm{~min}$ to $95^{\circ} \mathrm{C}$, and the supernatant was analyzed by SDS-PAGE and western blot. The polyclonal CathD antibodies were used to verify CathD precipitation, whereas polyclonal PLP antibodies were used to verify PLP precipitation and to detect bond CathD or VAMP7.

\section{Statistical analysis}

Statistical analysis was performed using GraphPad Prism 6.00 software (La Jolla, CA, USA). Data were expressed as mean \pm s.e.m. All groups were compared using two-tailed unpaired Student's $t$-test unless otherwise specified. $P<0.05$ indicated significant difference.

\section{RESULTS}

\section{CNS myelination is disrupted in CathD ${ }^{-1-}$ mice}

The myelin formation in the corpus callosum of $\mathrm{CathD}^{+/+}$and CathD $^{-/-}$mice was assessed on P11, P14 and P24 using the lipophilic dye Fluoromyelin Green and the immunostaining against PLP (red; Figure 1a). The positive signals for Fluoromyelin Green and PLP began to appear on P11 in the corpus callosum of both $\mathrm{CathD}^{-1-}$ and $\mathrm{CathD}^{+/+}$mice. However, such signals were much weaker in the CathD ${ }^{-/-}$mice than those in the CathD $\mathrm{D}^{+/+}$mice, suggesting a delay in the onset of myelination. On P14 and P24, a gradual increase in the density of Fluoromyelin Green staining and PLP immunofluorescence was observed in the corpus callosum of $\mathrm{CathD}^{+/+}$mice. However, the density of Fluoromyelin Green staining and PLP immunofluorescence in the corpus callosum of CathD ${ }^{-/-}$ mice was lower at each progressing time point and the increase in the density of Fluoromyelin Green staining and PLP immunofluorescence in the corpus callosum of the $\mathrm{CathD}^{-/-}$mice was much lower than that in the CathD ${ }^{+/+}$ mice. Interestingly, it was found that lots of PLP were located around the cell nuclei, by forming large punctas in the corpus callosum from P14 to P24 in CathD ${ }^{-/-}$mice, whereas finely distributed punctas along axons could be seen as lines in CathD ${ }^{+/+}$mice at the same time point. To assess PLP expression quantitatively during the corpus callosum development, western blot analysis was performed on brain samples at P11, P14 and P24 (Figure 1b). Consistent with the corpus callosum staining, age-related increases in terms of PLP expression were observed in CathD ${ }^{-1-}$ and the control mice. At P11, PLP expression was not readily detectable with western blot analysis. At P14 and P24, PLP expression in CathD ${ }^{+/+}$mice was twice as high as that in the CathD ${ }^{-/-}$mice (Figure $1 \mathrm{~b}$ and $\mathrm{c}$ ). Furthermore, the levels of $\mathrm{CNP}$ and Olig2, markers of $\mathrm{OL}$ specification and CNS myelination in the corpus callosum, were also examined. Compared with the $\mathrm{CathD}^{+/+}$mice, the expression level of CNP and Olig2 in P14 and P24 but not P11 in brain samples of the CathD ${ }^{-/-}$mice was lower (Figure 1b, d and e). These results indicated that CNS myelination was disrupted in the brain of CathD ${ }^{-/-}$mice.

\section{OL maturation is inhibited in the $\mathrm{CathD}^{-/-}$mice}

The dysmyelination in a CathD ${ }^{-/-}$mouse could be caused by the defect of proliferation, migration or maturation in OLs. The expression of Olig2 and CC1, a marker for post-mitotic oligodendrocytes, ${ }^{20}$ was examined in the corpus callosum of CathD ${ }^{+/+}$and CathD ${ }^{-/-}$mice at P11, P14 and P24 (Figure 2a and b). It was found that the density of Olig2+ cells was similar in the CathD ${ }^{-/-}$and CathD ${ }^{+/+}$mice at $\mathrm{P} 11$, but was reduced at P14 and P24. At P24, the decrease of Olig2+cell density was greater in the corpus callosum of the CathD ${ }^{-/-}$mice than that in the CathD ${ }^{+/+}$mice. On the other hand, as compared with the $\mathrm{CC} 1+$ cell density that continuously increased from P11 to P24 in the corpus callosum of CathD ${ }^{+/+}$mice, the $\mathrm{CC} 1+$ cell density in the CathD $\mathrm{D}^{-/-}$mice was not significantly different from that of the controls at P11 and only slightly increased from P14 to P24. It is worth noting that there was no difference in terms of Olig2+/CC1 - cells between CathD ${ }^{+/+}$ and CathD $^{-1-}$ mice at P11, P14 and P24, thus further demonstrating that the reduction of Olig2+cells in the CathD ${ }^{-/-}$mice was the reason for their different $\mathrm{CCl} 1+$ cell counts. Therefore, it is postulated that CathD deficiency leads to a selective loss of mature oligodendrocytes.

To identify apoptotic cells, the corpus callosum was immunostained with an antibody against activated caspase- 3 (Figure 3a). The numbers of activated caspase- $3+$ cells in the corpus callosum of $\mathrm{CathD}^{-/-}$mice and controls were not statistically different from P11 to P24 (Figure 3b), suggesting that the rates of apoptotic cell death were similar at each time point and apoptosis was not the main cause for the decreased mature oligodendrocytes. The development of OLs was further examined in vitro by culturing primary pure OPCs collected from the CathD ${ }^{+/+}$and CathD ${ }^{-/-}$mice. After culturing for 3 , 5,7 and 14 days in a differentiation medium, the mature oligodendrocytes were immunostained with the antibody against MBP, a marker for mature oligdendrocytes (Figure 4a). The percentage of total MBP+ mature oligodendrocytes in the CathD ${ }^{-/-}$mice continuously increased from 
a
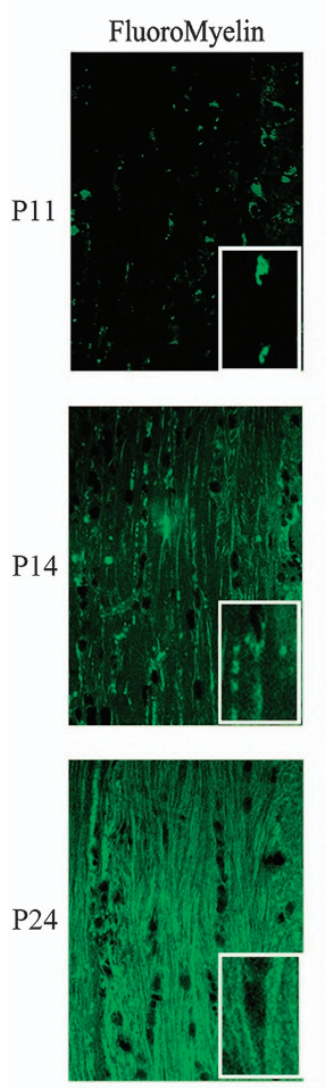

b
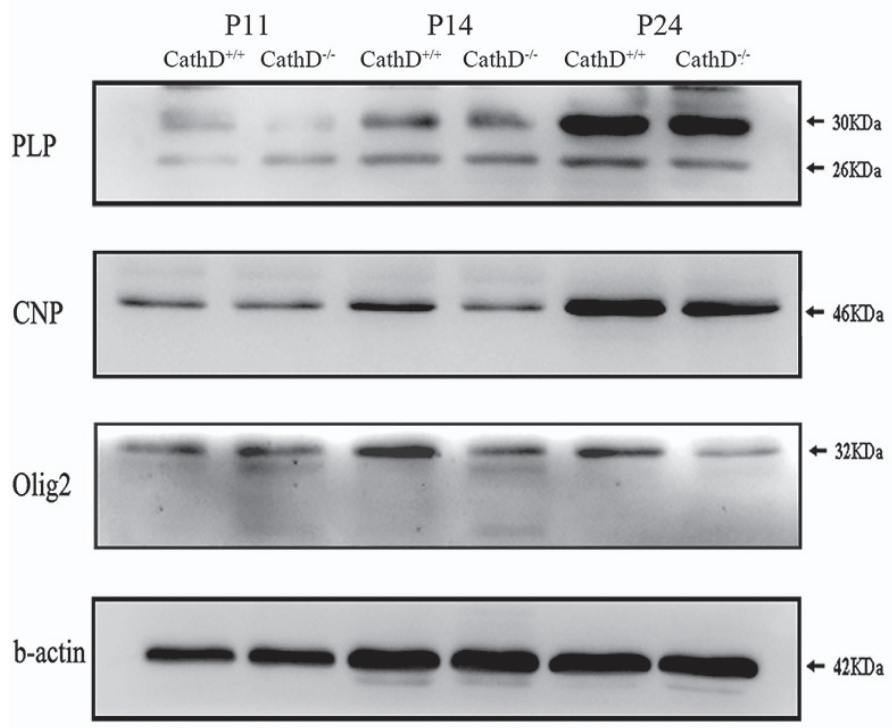

CathD ${ }^{+/+}$
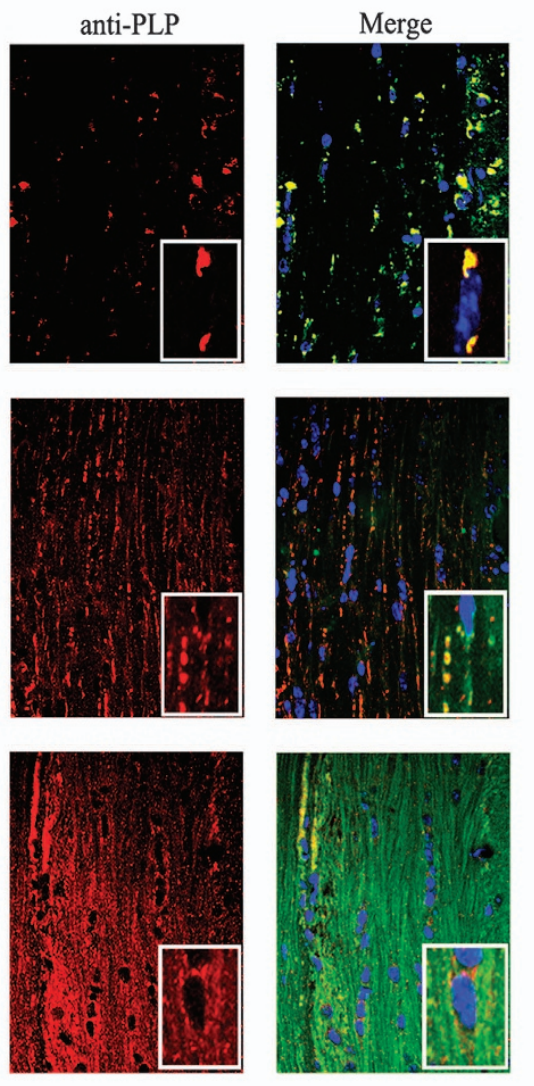
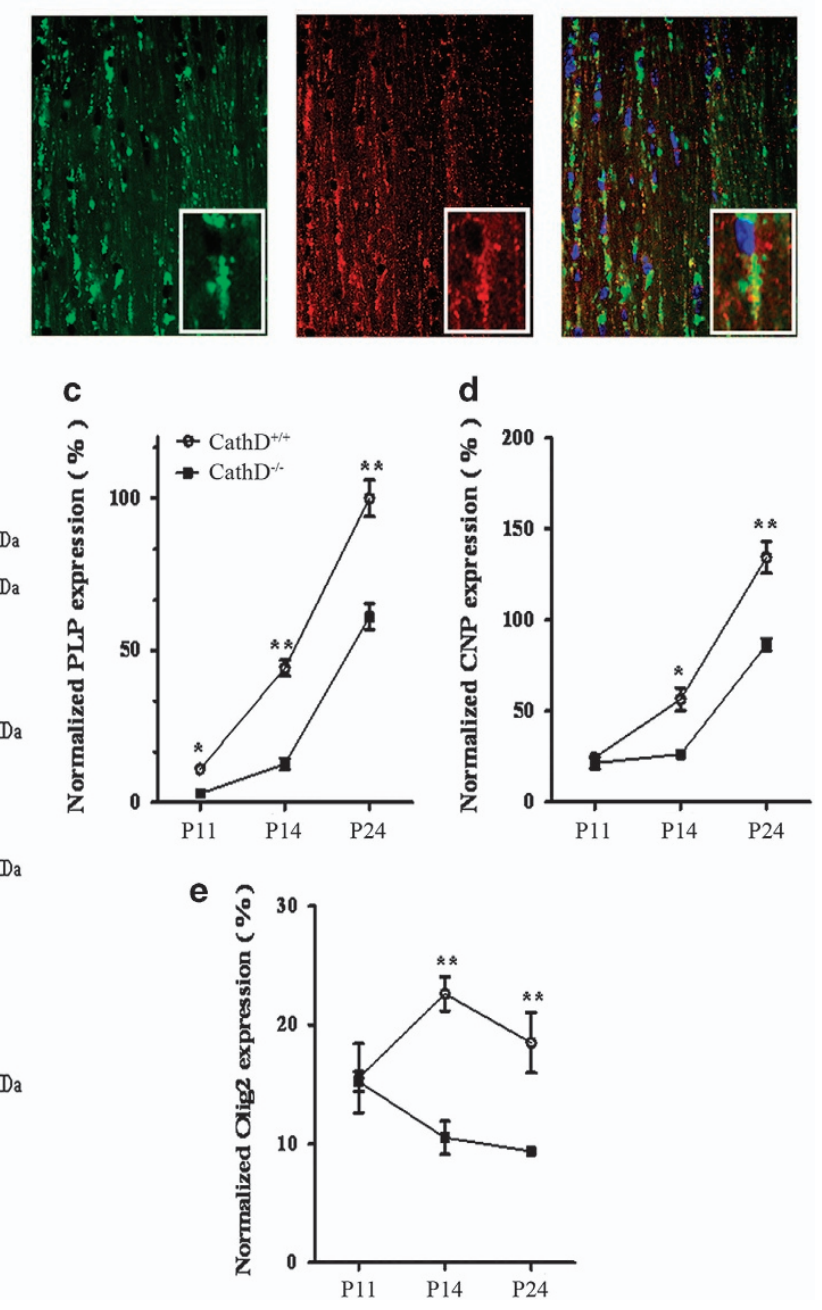

day 3 to day 14, but the increase was lower than that in the OPCs of CathD ${ }^{+/+}$mice. On day 14, the percentage of total $\mathrm{MBP}+$ mature oligodendrocytes was similar in both groups (Figure 4d). Furthermore, compared with the CathD ${ }^{+/+}$mice, the $\mathrm{MBP}+$ mature oligodendrocytes of $\mathrm{CathD}^{-/-}$mice displayed simpler morphologies with fewer membranes at each time point (Figure $4 \mathrm{~b}, \mathrm{c}$ and e), suggesting that CathD deficiency inhibited oligodendrocyte maturation. 
Figure 1 CNS myelination was disrupted in CathD ${ }^{-1-}$ mice. (a) Representative coronal sections of the corpus callosum from P11 (top), P14 (middle) and P24 (bottom) mice demonstrated increasing PLP (red) expression and Fluoromyelin Green staining intensity with age in CathD ${ }^{+/+}$and CathD ${ }^{-/-}$mice. At all ages, both PLP immunostaining and Fluoromyelin Green staining were weaker in the CathD ${ }^{-/-}$mice. The inner box showed that more PLP punctas were localized in the body of cells in the CathD ${ }^{-/-}$mice compared with that in CathD ${ }^{+/+}$ mice. Scale bar, $20 \mu \mathrm{m}$. (b) Western blot of developmental brain protein expression indicated that, at each stage of age, a lower level of CNS myelin proteins such as PLP and CNP was expressed in the CathD ${ }^{-/-}$brain as compared with that in the CathD ${ }^{+/+}$brain, although the expression level of these proteins increased with age in both groups. The expression level of Olig2 was the highest at P14 in the CathD +/+ mice and decreased with further brain development. However, Olig2 expression was lower at P14 and P24 in the CathD ${ }^{-/-}$mice as compared with that in the CathD ${ }^{+/+}$mice (c-e). Quantification of western blot data showed that, after normalization to actin expression, the expression of PLP, CNP and Olig2 at P14 and P24 was significantly lower in the CathD ${ }^{-1-}$ mice as compared with that in the controls $\left(n=3 ;{ }^{*} P<0.05,{ }^{* *} P<0.01\right)$. CathD, cathepsin D; CNS, central nervous system; PLP, proteolipid protein.

a
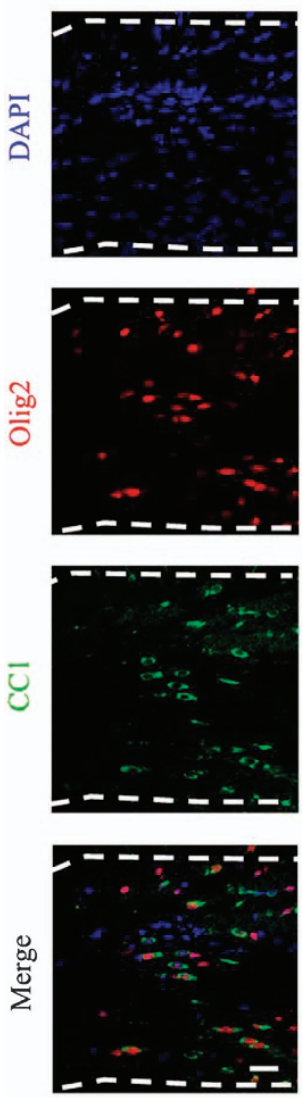

$\mathrm{CathD}^{-/}$
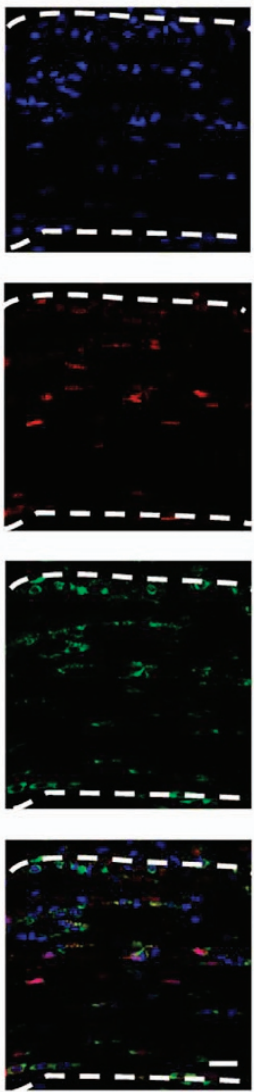

b
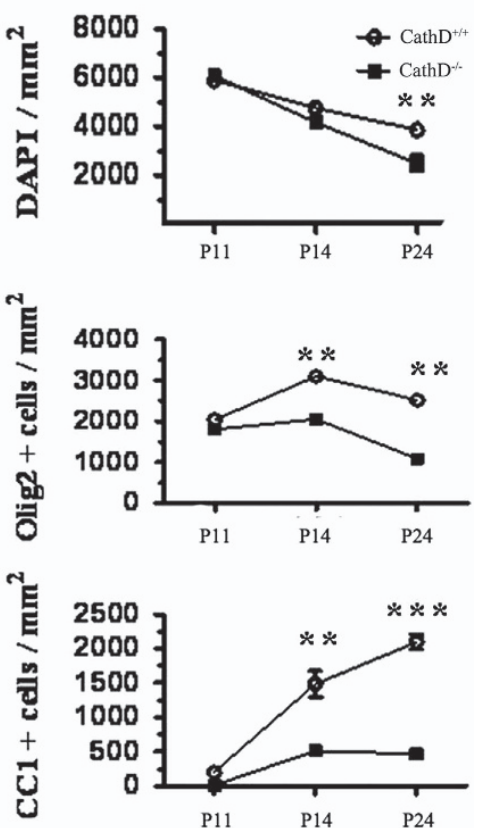

沓

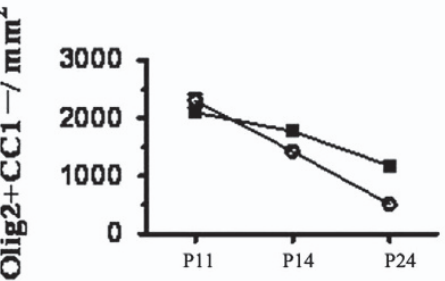

Figure 2 The number of mature oligodendrocytes was decreased in the corpus callosum of CathD ${ }^{-1-}$ mice. (a) Immunohistochemical analysis of coronal sections of the corpus callosum. OLs were labeled with antibodies against Olig2 (red) and CC1 (green) in the corpus callosum of P14 CathD ${ }^{+/+}$and CathD ${ }^{-/-}$mice. Nuclei were counterstained with DAPI (blue). The decreased number and the round morphology of $\mathrm{CC} 1+$ cells were observed in the corpus callosum of CathD ${ }^{-1-}$ mice, as compared with the linear chains of elongated $\mathrm{CC} 1+$ in the corpus callosum of CathD ${ }^{-1-}$ mice. (b) Quantification of cell types in the corpus callasum at P11, P14 and P24. The number of $\mathrm{CC} 1+$ cells reached a plateau in the CathD ${ }^{-1-}$ mice at $\mathrm{P} 14$ but continued to increase in the corpus callosum of CathD ${ }^{+/+m i c e}$. Average $\pm \mathrm{S}$. e.m. was obtained from three to six pairs of CathD $D^{+/+}$and CathD ${ }^{-/-}$mice at each time point. ${ }^{* *} P<0.01,{ }^{* *} P<0.001$. Scale bar, $20 \mu \mathrm{m}$. CathD, cathepsin D; DAPI, 4, 6-diamidino-2-phenylindole dihydrochloride; OL, oligodendrocyte lineage cell.

More PLP accumulates in LEs/Ls of OLs in CathD ${ }^{-1-}$ mice PLP is initially targeted to LEs/Ls by using a cholesteroldependent and clathrin-independent endocytosis pathway, and then transported from LEs/Ls to the plasma membrane and myelin membrane upon activation by neuronal cells. ${ }^{21}$ To analyze whether CathD deficiency had an impact on the subcellular localization of PLP, the intracellular localization of PLP and LAMP1, a lysosome marker, was observed in vitro (Figure 5a). Using confocal microscopy in conjunction with Z-stack, it was discovered that the OPCs of $\mathrm{CathD}^{+/+}$mice 
a

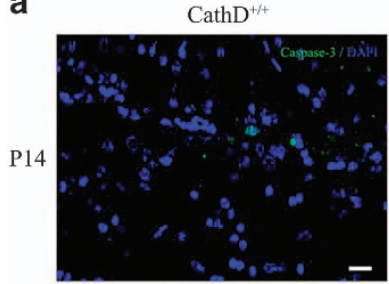

CathD

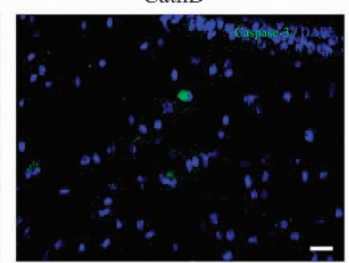

b

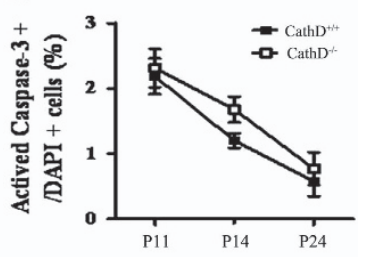

Figure 3 CathD deficiency did not lead to significantly increased apoptosis. (a) Coronal sections of P14 corpus callosum immunolabeled for activated caspased-3 (green) demonstrated no significantly increased apoptosis in the CathD-/- mice, as compared with those of the CathD+/+ mice. (b) Quantification of the density of activated caspased-3+ cells in the corpus callosum of CathD $-/-$ and CathD $+/+$ mice at P11, P14 and P24. The number of apoptotic cells was normalized to the surface area of the corpus callosum $(n=3)$. Scale bar, $20 \mu \mathrm{m}$. CathD, cathepsin D.

a

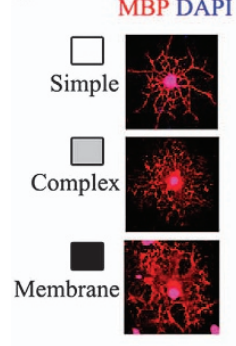

b

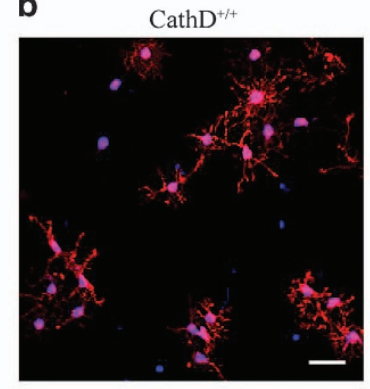

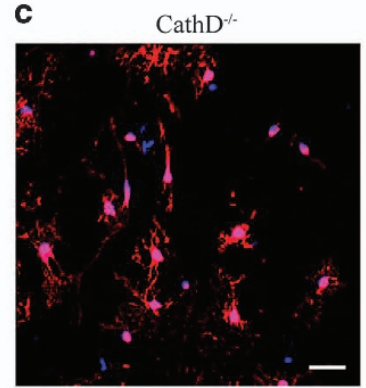

5 DIV d

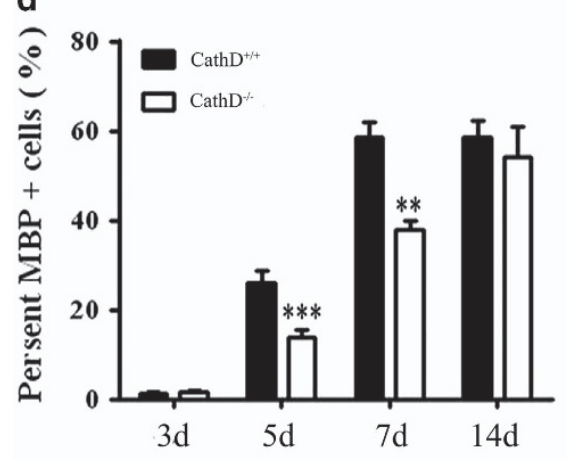

e

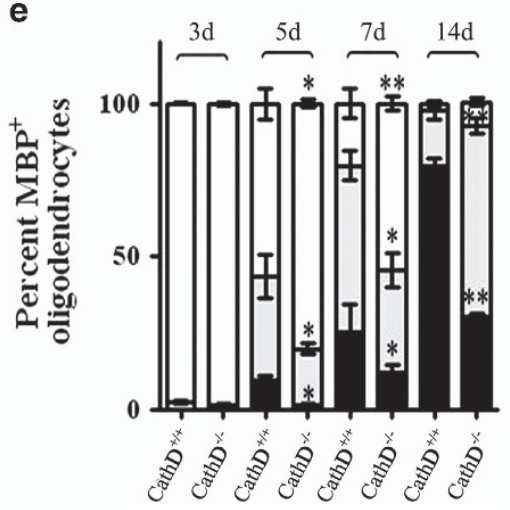

Figure 4 Inhibition of CathD functions delayed oligodendrocyte maturation. (a-c) OPCs of CathD+/+ and CathD - /- mice were cultured in a differentiation medium and were immunolabeled with antibodies against MBP (red) on day 5 in vitro. (a) Morphological criteria for the maturation state of differentiating OLs included simple, complex or membrane morphologies. Compared with cells obtained from the CathD $+/+$ mice (b), cells from the CathD - / - mice contained fewer mature oligodendrocytes and a decreased number of processes associated with MBP+ mature oligodendrocytes. Scale bar, $20 \mu \mathrm{m}$. (d, e) Statistical chart for the percentage of MBP+ cells cultured for 3d, $5 d, 7 d$ and $14 \mathrm{~d}$ in the differentiation medium. (d) Cells from the CathD-/ - mice showed a decreased proportion of MBP+oligodendrocytes at the beginning of differentiation, but no difference was observed on day 14. (e) As compared with cells from the CathD+/+ mice, the cells from the CathD-/ - mice showed an increased proportion of MBP+ oligodendrocytes with simple morphologies and a decreased proportion of $\mathrm{MBP}+$ oligodendrocytes with complex membrane morphologies. ${ }^{*} P<0.05,{ }^{*} P<0.01,{ }^{* *} P<0.001$. CathD, cathepsin $\mathrm{D}$; d, day; $\mathrm{MBP}$, myelin basic protein; OPC, oligodendrocyte precursor cell.

started to express PLP during the first 2 day of culture in the proliferation medium, and the colocalization of PLP and LAMP1 was observed in $\sim 90 \%$ of the cell bodies. However, little colocalization of PLP and LAMP1 in the processes of OLs was observed after $24 \mathrm{~h}$ in the differentiation medium. On the other hand, in the OPCs of CathD ${ }^{-1-}$ mice, PLP accumulated in the LEs/Ls of both the cell bodies and processes of OLs, where the colocalization of PLP and LAMP1 was nearly $\sim 100 \%$. Meanwhile, it was found that a fewer number of processes were present in the OLs of CathD ${ }^{-1-}$ mice than those of the CathD ${ }^{+/+}$mice after $24 \mathrm{~h}$ of culturing in the differentiation medium, suggesting that the deficiency of CathD not only disturbed PLP distribution, but also inhibited oligodendrocyte maturation.

Similar results were obtained in vivo. Significant colocalization of PLP and LAMP1 was observed in the corpus callosal cells of CathD ${ }^{+/+}$mice at P11 and such colocalization was increased by twofold in the P14 mice but decreased 
a
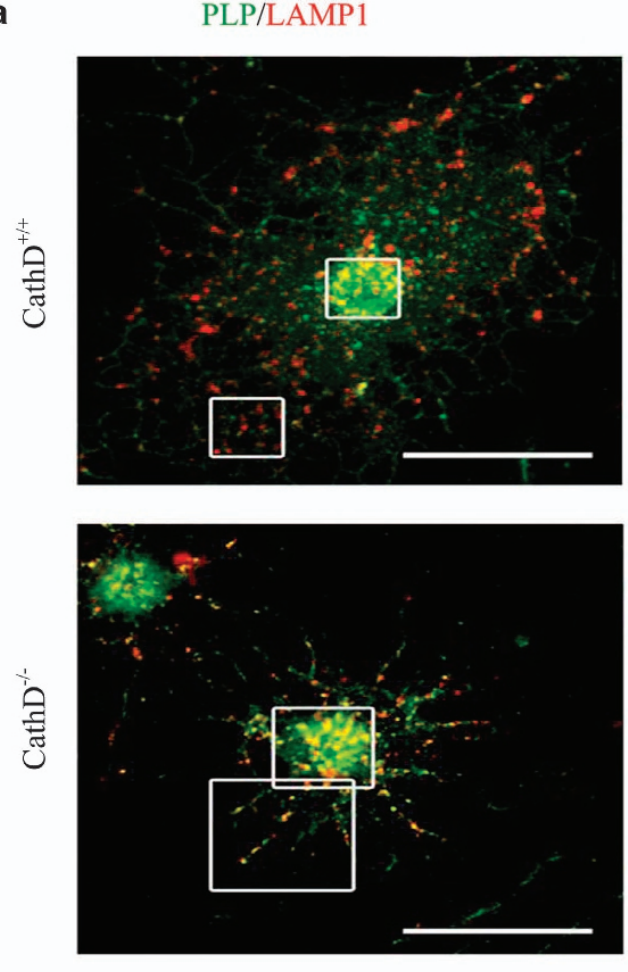

b
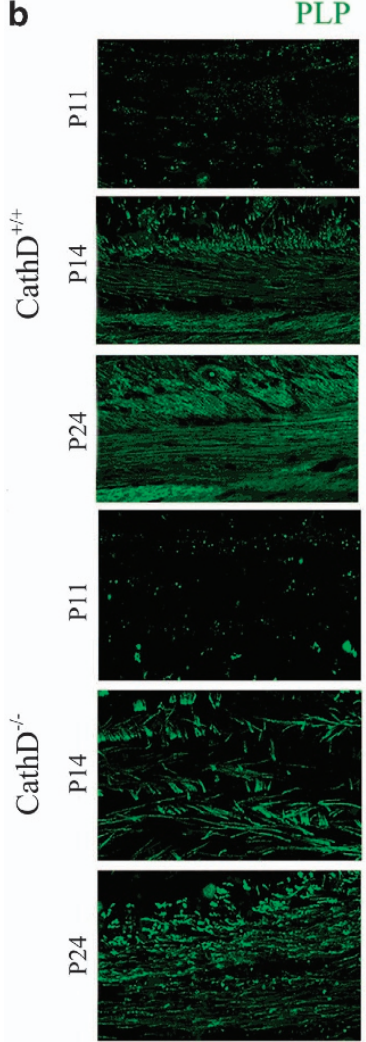
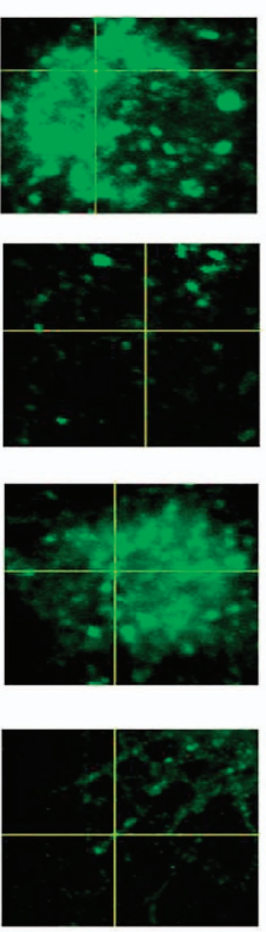

LAMP1
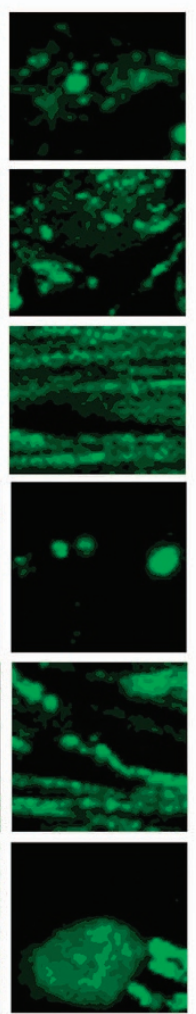
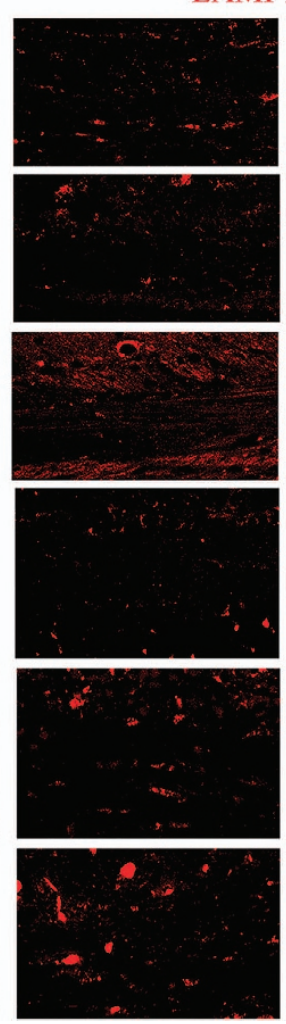
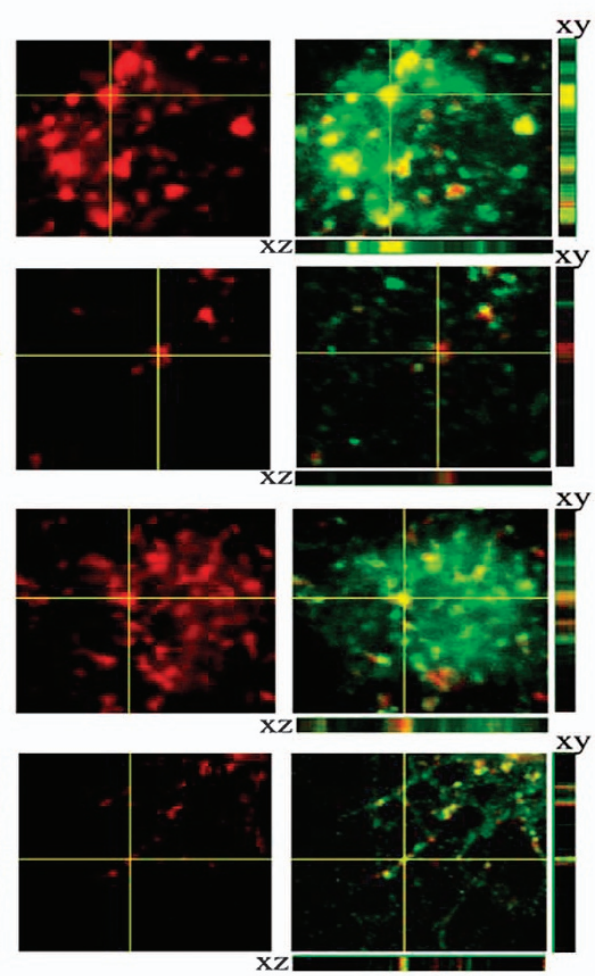

MERGE
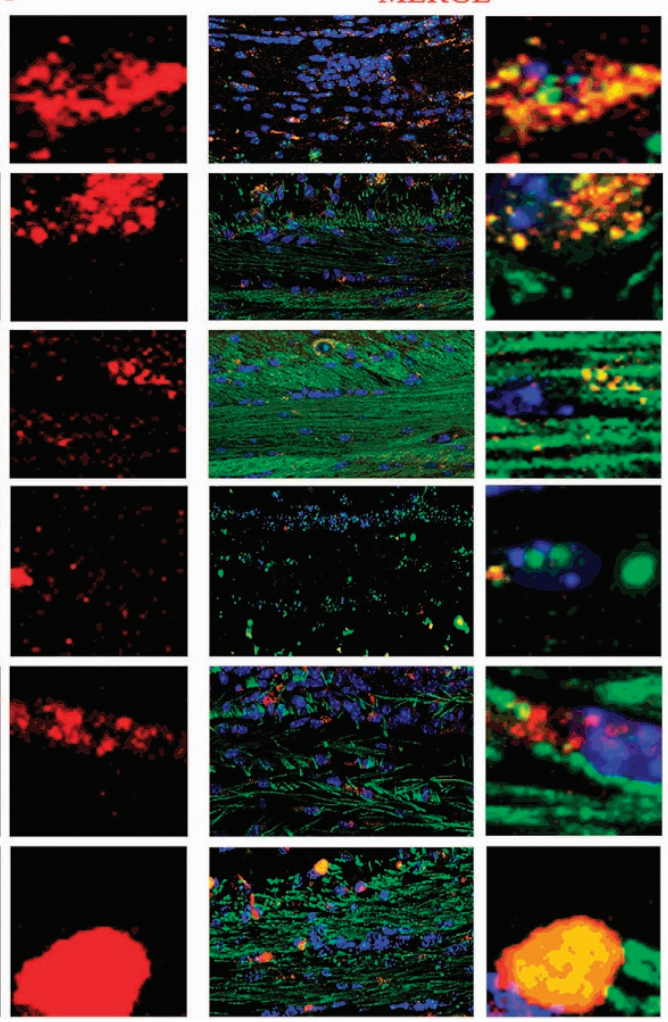

in the P24 mice (Figure 5b). In contrast to the CathD ${ }^{+/+}$mice, only a little expression of PLP was present in the corpus callosal cells of CathD ${ }^{-/-}$mice at P11 and the colocalization of PLP and LAMP1 started to increase at P14. Colocalization

at a level close to $100 \%$ was noted for the PLP and LAMP1 in the corpus callosum cells of CathD ${ }^{-/-}$mice, which also formed larger punctas than those in the controls (Figure 5b). 
Figure 5 More PLP accumulates in the LEs/Ls of OLs in the CathD ${ }^{-/-}$mice. (a) Confocal immunofluorescence microscopy in conjunction with z-stack demonstrated the colocalization of endogenous PLP (green) with LAMP1 (red) in the OLs of CathD ${ }^{-1-}$ and CathD+/+ mice. In addition, compared with the controls, more PLP-EGFP signals colocalized with LysoT signals when the cells were treated with antipain. Top, OLs of CathD ${ }^{+/+}$mice; bottom, OLs of CathD ${ }^{-/-}$mice. (b) Immunohistochemistry results showed the localization of PLP in the LEs/Ls on the brain coronal sections from P11, P14 and P24 CathD ${ }^{+/+}$and CathD ${ }^{-/-}$mice. In the corpus callosal cells of CathD ${ }^{+/+}$mice, significant colocalization of PLP (green) and LAMP1 (red) was observed at P11, and the colocalization was increased by twofold in P14 mice and decreased in P24 mice. On the other hand, little expression of PLP and colocalization between PLP and LAMP1 was seen in the corpus callosal cells of CathD ${ }^{-1-}$ mice at P11, although the colocalization started to increase at P14 and reached $\sim 100 \%$ at P24. Scale bar, $20 \mu \mathrm{m}$. CathD, cathepsin D; OL, oligodendrocyte lineage cell; LE/L, late endosome/lysosome; PLP, proteolipid protein.
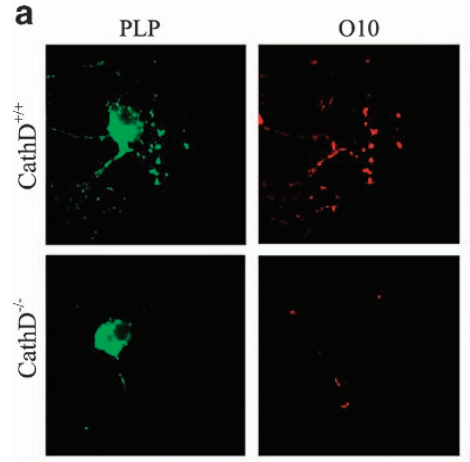

e

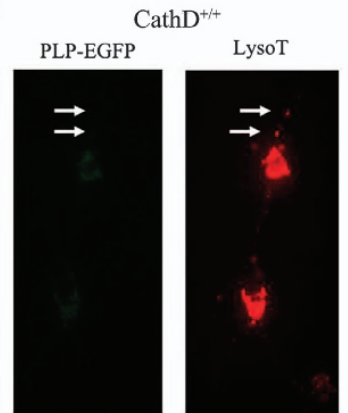

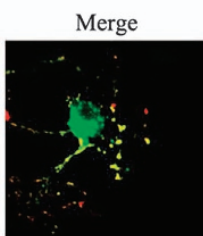
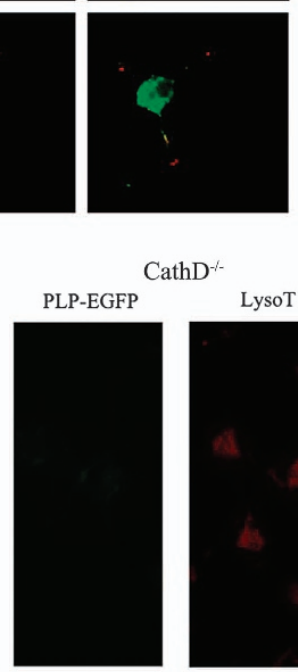

$\mathrm{CathD}^{-/-}$

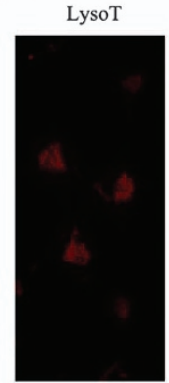

b
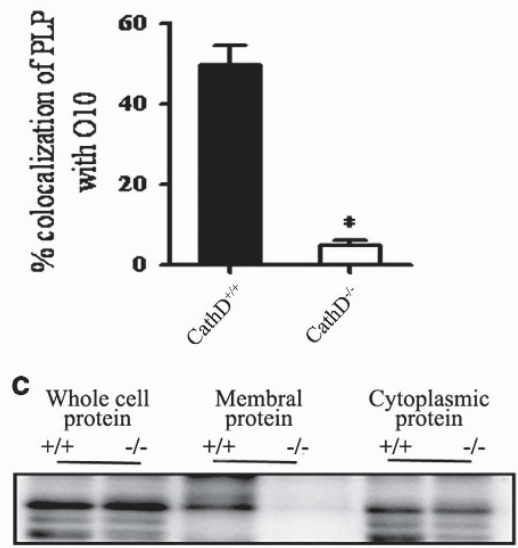

d

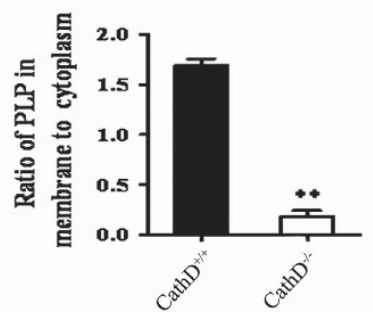

Figure 6 Fewer PLPs were distributed on the plasma membrane of OLs in the CathD ${ }^{-1-}$ mice. (a) Surface staining of living cells with 010 $\mathrm{mAb}$ at $4{ }^{\circ} \mathrm{C}$ showed that the minority of PLP was found on the surface of OLs from the CathD ${ }^{-/-}$mice. (b) Quantitative analysis of the colocalization of PLP with 010. Error bars represent s.e.m. $(n>20)$. (c) Biotinylation labeling of the PLP on cell surface. $+/+$, CathD $+/+$ mice; -I-, CathD ${ }^{-/}$mice. Western blots were performed for biotinylated (membrane-bound PLP) and nonbiotinylated protein (cytoplasmic PLP) fractions, as well as for whole-cell fractions (whole-cell PLP); the blots were probed with rabbit anti-PLP polyclonal antibodies. Compared with OLs from the CathD ${ }^{+/+}$mice, the OLs from the CathD ${ }^{-/-}$mice were associated with a decreased ratio of biotinylated (membrane-bound PLP) to nonbiotinylated PLP (cytoplasmic PLP). There was no significant difference in terms of the total cell PLP expression between the CathD ${ }^{+/+}$and the CathD ${ }^{-/-}$mice. (d) Quantitative analysis of c showed the ratio of membrane-bound PLP to cytoplamic PLP $(n=5)$. (e) TIRFM images demonstrated that few or no PLP-EGFP that contained vesicles was present in the proximity $(100 \mathrm{~nm})$ of the plasma membrane of OLs from the CathD ${ }^{-1-}$ mice. ${ }^{*} P<0.05,{ }^{*} P<0.01$; scale bar, $20 \mu \mathrm{m}$. CathD, cathepsin $\mathrm{D}$; OL, oligodendrocyte lineage cell; PLP, proteolipid protein; TIRFM, total internal reflection fluorescence microscopy.

Less PLP distribution on the plasma membrane of OLs in CathD $^{-/-}$mice

Next, experiments were carried out to examine whether the developmental regulation of PLP distribution between LEs/Ls and the plasma membrane of OLs was disturbed by the deficiency of CathD. Surface staining showed that, in the differentiation medium, fewer PLP was located at the plasma membrane of cells in the CathD ${ }^{-/-}$mice than those in the CathD ${ }^{+/+}$mice (Figure 6a); quantitative analysis showed that only $5 \%$ of O10 mAb colocalized with PLP in the OLs of CathD ${ }^{-/-}$mice as compared with $49.7 \%$ in the CathD ${ }^{+/+}$mice
(Figure 6b). Biotinylation labeling of cell surface proteins showed that, compared with cells of the CathD ${ }^{-/-}$mice, the cells of $\mathrm{CathD}^{+/+}$mice were associated with a decreased ratio of biotinylated (membrane-bound) to nonbiotinylated (cytoplasmic) PLP (Figure 6c). There were no significant alternations between the $\mathrm{CathD}^{+/+}$and $\mathrm{CathD}^{-/-}$mice in terms of total cell PLP expression. Densitometry measurements of the PLP bands showed that the ratio of membrane bound to cytoplasmic PLP was $1.69 \pm 0.04$ in the CathD ${ }^{+/+}$mice, and this ratio decreased to $0.18 \pm 0.04$ in the $\mathrm{CathD}^{-1-}$ mice (Figure 6d). 
Subsequently, TIRFM was used to analyze the behavior of the vesicle pool containing peripheral PLP. It was observed that many PLP-EGFP containing LAMP1-positive vesicles localized within the 100-nm vicinity of the plasma membrane in both the cell bodies and processes of living OLs of the CathD ${ }^{+/+}$ mice. In contrast, few or no PLP-EGFP that contained LAMP1positive vesicles was seen in the proximity of plasma membrane of the living OLs from the CathD ${ }^{-/-}$mice (Figure 6e). These results suggested that fewer PLP appeared on the plasma membrane of OLs in the CathD ${ }^{-/-}$mice.

The transport of PLP from LEs/Ls to the plasma membrane of OLs slowed down in the CathD ${ }^{-1-}$ mice

Two possibilities may lead to the abnormal distribution of PLP between the LEs/Ls and the plasma membrane of OLs in the CathD ${ }^{-1-}$ mice. One possibility is that fewer PLPs were transported out of LEs/Ls to the plasma membrane of OLs, whereas the other one is that more PLPs were endocytosed and taken up in LEs/Ls. To analyze the putative exocytic trafficking of PLP from LEs/Ls, live cell imaging experiments were performed using LysoT in the PLP-EGFPexpressing OLs from the CathD ${ }^{+/+}$and $\mathrm{CathD}^{-/-}$mice. A high degree of colocalization between PLP-EGFP and LysoT was present in the perinuclear and peripheral pools of OLs collected from both types of mice, similar to the immunocytochemistry results related to PLP and LAMP1. The analysis regarding the dynamics of PLP-EGFP/LysoT-positive vesicles revealed that, in the OLs from $\mathrm{CathD}^{-/-}$mice, most PLPcontaining $\mathrm{LE} / \mathrm{L}$ vesicles moved more slowly toward the plasma membrane at the distal end of the processes than those in the CathD ${ }^{+/+}$mice (Figure 7a). A quantitative analysis revealed that, as compared with OLs from the CathD ${ }^{+/+}$mice, fewer PLP-EGFP/LysoT-positive vesicles were mobile in the OLs from the CathD ${ }^{-1-}$ mice $(26.68 \pm 1.98$ versus $6.27 \pm 0.35 \%$; Figure 7b).

To test the latter hypothesis described above, endocytosis experiments were performed using the $\mathrm{O} 10 \mathrm{mAb}$. There was no significant difference in terms of the uptake of O10 in OLs from the CathD ${ }^{-/-}$and the CathD ${ }^{+/+}$mice (Figure $7 \mathrm{c}$ and $\mathrm{d}$ ). Thus, neither clatherin-dependent nor clatherin-independent endocytosis of PLP seemed to be the underlying mechanism for the accumulation of PLP in the LEs/Ls of OLs from the CathD $^{-1-}$ mice.

\section{VAMP7 may act as the mediator between CathD and PLP}

Confocal imaging revealed that CathD was colocalized with PLP in the perinuclear regions and processes of OLs (Figure 8a). Immunoelectron microscopy showed that gold particles marked CathD (larger, $15 \mathrm{~nm}$ particles) and PLP (smaller, $5 \mathrm{~nm}$ particles) colocalized in large vesicles inside LEs/Ls (Figure 8b). In vitro pull-down assay showed that PLP-myc was recovered from immunoprecipitation of CathD (Figure 8c). Subsequently, co-immunoprecipitation experiments were carried out to determine whether endogenous CathD also bond native PLP. Under non-denaturing conditions, antibodies against CathD precipitated PLP isoforms in the immune complexes. In the brain of a P20 mouse, a 30-kDa PLP-bound complex was associated with CathD and was found in the immunoprecipitate, whereas a $26-\mathrm{kDa}$ DM20-bound complex was not associated with CathD and was not found in the immunoprecipitate. No PLP was detected in a control immunoprecipitation experiment that used an irrelevant IgG (Figure 8d). In the reciprocal co-immunoprecipitation experiments using a goat anti-PLP antibody, interestingly, only the intermediate $48-\mathrm{kDa}$ and the mature light-chain $14 \mathrm{kDa}$ of CathD were found to obviously co-precipitate with the PLP in the immune complex, whereas a $52-\mathrm{kDa}$ pro-CathD and $34-\mathrm{kDa}$ mature heavy chains did not co-precipitate with the PLP in the immune complex (Figure 8e). Confocal imaging experiments revealed that PLP/ VAMP7 and CathD/VAMP7 colocalized in a high degree (Figure 8f). In addition, in a co-immunoprecipitation experiment using goat anti-PLP antibody, VAMP7 was found to significantly co-precipitate with the PLP in the immune complex (Figure 8g).

\section{DISCUSSION}

\section{CathD and myelination}

In peripheral nervous system, an important event before myelination is the precise arrangement of Schwann cells along the nerves. Initially, immature Schwann cells encircle a group of nerves. Subsequently, individual cells migrate away from the cohort and start to find the axons to the periphery of the nerve bundle, where a 1:1 relationship with the nerve bundle was adopted. ${ }^{21}$ Unlike myelination of peripheral nervous system by Schwann cells, myelination of CNS is a more complex process and begins with the specification of proliferating and migratory OPCs, followed by the differentiation of these cells into postmitotic oligodendrocytes that myelinate multiple axons. These processes must be carried out for a large number of different axons. ${ }^{22}$ Hence, a defect in any one of these steps could cause a severe dysmyelination of CNS.

In human, CathD is a protein encoded by the CTSD gene. As a member of the peptidase A1 family, CathD is an aspartic endoprotease and is ubiquitously distributed in lysosomes. CathD accounts for $11 \%$ of all proteolytic enzymes in lysosomes. During its transportation to lysosomes, the $52-\mathrm{kDa}$ pro-CathD is proteolytically processed to form a 48 $\mathrm{kDa}$ single-chain intermediate that is an active enzyme located in the endosomes. Further proteolytic processing yields a mature and active lysosomal protease, which is composed of both heavy $(34 \mathrm{kDa})$ and light $(14 \mathrm{kDa})$ chains. $^{23}$ The main functions of CathD include digestion of proteins and peptides in acidic lysosomes, and participation in biological processes such as cellular protein renewal and tissue homeostasis. ${ }^{24}$ CathD deficiencies are fatal neurological diseases that are characterized by a significant loss of neurons and myelin in human infants and sheep. ${ }^{25}$ CathD knockout mice also show pronounced myelin changes in the brain, and myelin-related PLP and MBP are both markedly reduced on postnatal day 24, and the amount of lipids characteristically high in myelin are significantly lowered as compared with the controls. ${ }^{11}$ 

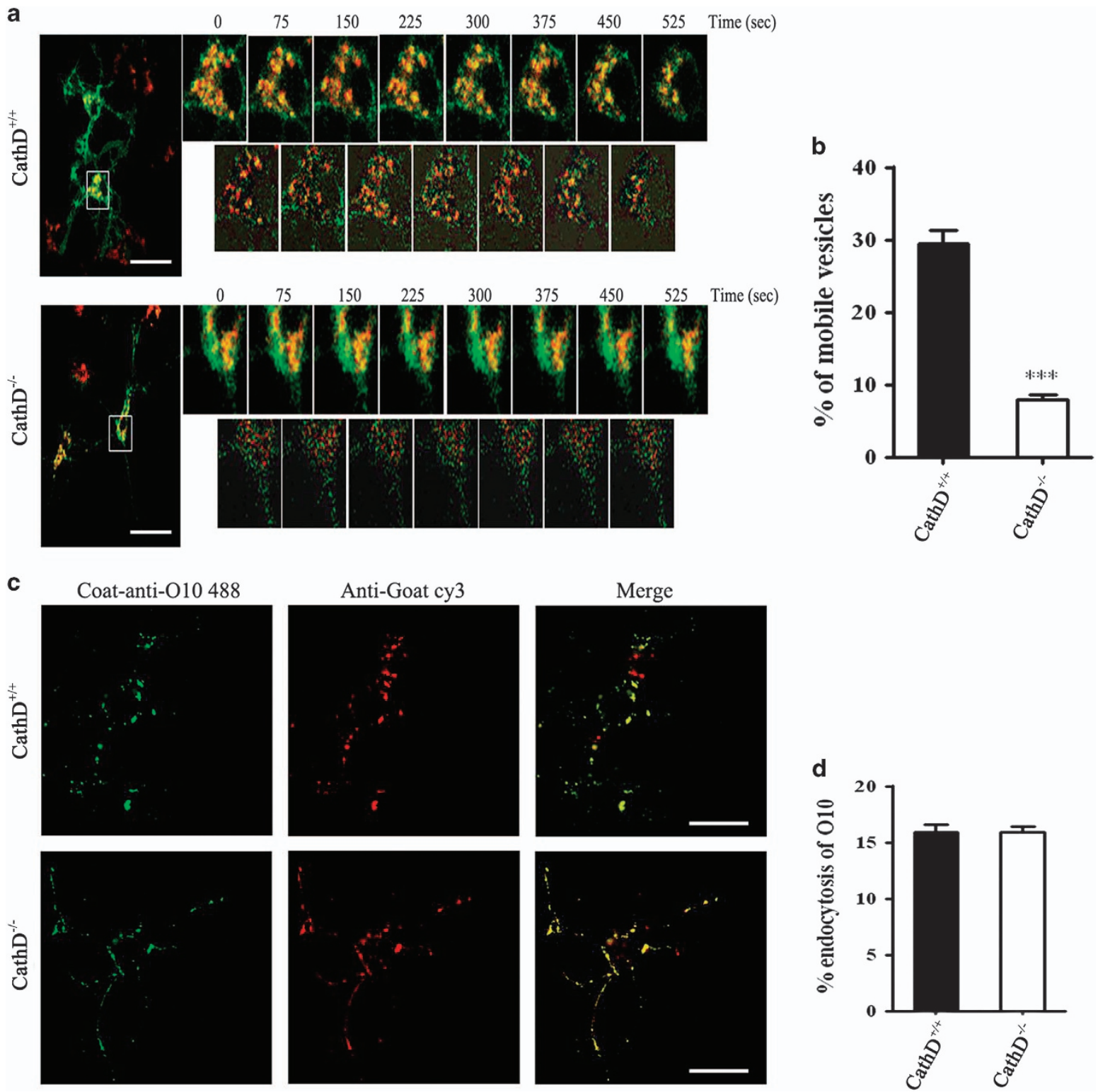

Figure 7 The transport of PLP from LEs/Ls to the plasma membrane of OLs slowed down in the OLs of CathD ${ }^{-/-}$mice. (a) Live cell imaging experiments of LysoT in PLP-EGFP expressing OLs from the CathD ${ }^{+/+}$and CathD ${ }^{-/-}$mice. PLP-EGFP (green) and LysoT markers of $\mathrm{LE} / \mathrm{Ls}$ (red) colocalized well in the OLs of the CathD ${ }^{+/+}$(top) and CathD ${ }^{-/-}$(bottom) mice. Compared with OLs of the CathD ${ }^{+/+}$mice, the colocalization of PLP-EGFP and LysoT decreased more quickly. Scale bar, $20 \mu \mathrm{m}$. (b) Statistical analysis regarding the mobility of PLPEGFP and LysoT-positive vesicles. Vesicle movement was analyzed by subtracting from each image the information in its preceding image of a time stack. The different image stack generated from the above operation was used to identify vesicle motility events. The data showed that the percentage of mobile vesicles was $26.68 \pm 1.98 \%(n=15)$ in the OLs of CathD ${ }^{+/+}$mice and $6.27 \pm 0.35 \%(n=18)$ in the OLs of CathD ${ }^{-1-}$ mice. ${ }^{* * *} P<0.01$. (c, d) Endocytosis experiments using $010 \mathrm{mAb}$ showed that neither clatherin-dependent nor clatherin-independent endocytosis of PLP seemed to be the underlying mechanism for the accumulation of PLP in LEs/Ls of OLs from the CathD ${ }^{-/-}$mice. (c) Goat-anti-010 488 (left, green) labeled the endocytic and non-endocytic vesicles in the OLs of CathD ${ }^{+/+}$and CathD ${ }^{-/-}$ mice. Anti-Goat Cy3 (middle, red) labeled the non-endocytic vesicles. The merged signal (right, yellow) represented the non-endocytic vesicles. Scale bar, $20 \mu \mathrm{m}$. (d) Statistical analysis showed the percentage of endocytic 010 ( $n=18$, respectively). CathD, cathepsin D; LE/ $\mathrm{L}$, late endosome/lysosome; OL, oligodendrocyte lineage cell; PLP, proteolipid protein.

However, few studies have investigated the myelination and the mechanisms underlying the defects of myelin sheaths in CathD ${ }^{-1-}$ mice.

Our data demonstrated that the formation of myelin in the CathD ${ }^{-/-}$mice was delayed as compared with the CathD ${ }^{+/-}+$ mice. Although the myelin content (as was indicated by PLP expression levels and Fluoromyelin Green staining) in the corpus callosum of the CathD ${ }^{-/-}$mice increased from P11 to $\mathrm{P} 24$, the extent of myelination in the $\mathrm{CathD}^{-/-}$mice was lower than that in the CathD ${ }^{+/+}$mice. However, CathD ${ }^{-/-}$ 

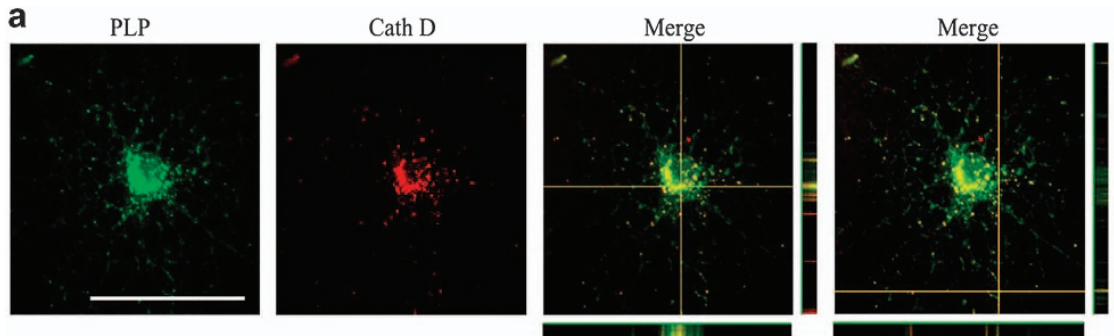

b

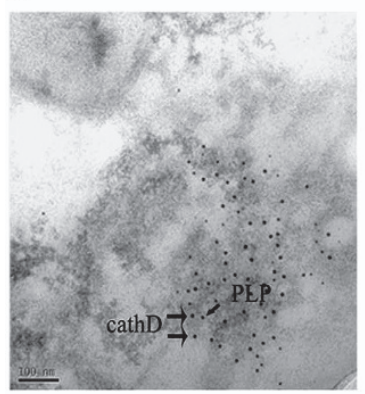

f

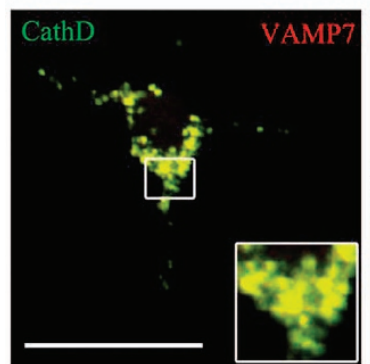

C
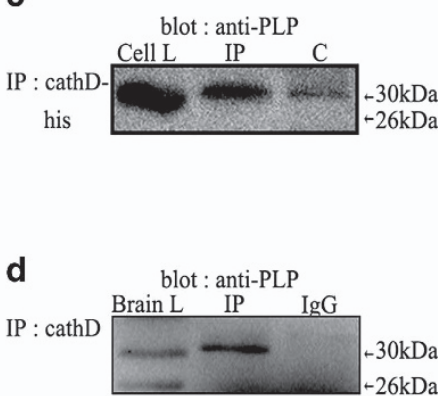

$+30 \mathrm{kDa}$

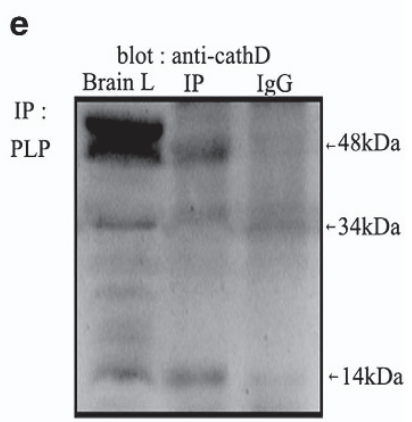

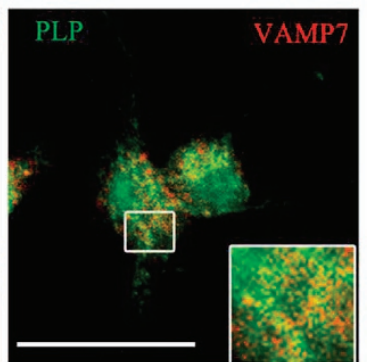

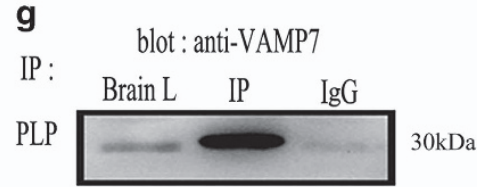

Figure 8 CathD may regulate the transport of PLP from LEs/Ls to the plasma membrane by binding to VAMP7 and PLP. (a) Confocal imaging experiments revealed that CathD was colocalized with PLP in the perinuclear regions and processes of OPCs. (b) Immunoelectron microscopy results of the P20 C57BL/6 mouse brain. Gold particles marked CathD (larger, $15 \mathrm{~nm}$ particles) and PLP (smaller, $5 \mathrm{~nm}$ particles) colocalized in large vesicles inside LEs/Ls. (c) Co-expressed CathD and myc-tagged PLP in Hek293 cells of an in vitro pull-down assay. Myc-PLP was recovered from the immunoprecipitation of CathD. (d) Under non-denaturing conditions, antibodies against CathD precipitated PLP isoforms in the immune complexes. (e) In the reciprocal co-immunoprecipitation experiments using a goat anti-PLP antibody, only the intermediate $48-\mathrm{kDa}$ and the mature light-chain $14 \mathrm{kDa}$ of CathD were found to obviously co-precipitate with the PLP in the immune complex, whereas 52-kDa pro-CathD and 34-kDa mature heavy chains did not co-precipitate with the PLP in the immune complex. (f) Confocal imaging experiments revealed that PLPNAMP7 and CathD/NAMP7 colocalized to a high degree. (g) VAMP7 was found to significantly co-precipitate with the PLP in the immune complex. Scale bar, $20 \mu \mathrm{m}$. CathD, cathepsin D; LE/L, late endosome/ lysosome; OPC, oligodendrocyte precursor cell; PLP, proteolipid protein.

mice showed normal development during the first 2 weeks but stopped in the third week. Eventually, they died on day $25 \pm 1$ in a state of anorexia due to widespread intestinal necrosis, thromboemboli, fulminant loss of $\mathrm{T}$ and $\mathrm{B}$ cells, and massive destruction of the thymus and spleen. Therefore, it is difficult to investigate the changes of myelination causing by CathD deficiency. Conditional CathD knockout mice may be useful to achieve further elucidation.

\section{CathD and OL development}

In the CNS, OLs develop from OPCs. OPCs can differentiate into oligodendrocytes, type II astrocytes and neurons in the brain, and are now considered as adult stem cells. ${ }^{26}$ Many known factors can regulate the differentiation of OPCs, including SOX10 and Hes5. ${ }^{27,28}$ There are also many factors regulating the maturation of OLs, including LINGO-1 and PSA-NCAM. ${ }^{29,30}$ However, no report has shown that lysosomal hydrolysis is related to OL development.

Several studies have shown that the activity and localization of CathD can be changed because of aging or different pathophysiological conditions. In patients with Alzheimer's disease, the activity of CathD increases markedly and can degrade beta amyloid, a soluble human brain extract under $\mathrm{pH}$ $4-6 .{ }^{31}$ At the time of cell death, mature CathD is present in the nucleus and is involved in regulating the proteolytic activation of endonuclease. ${ }^{32}$ In tumors, part of CathD fragments (amino acids 27-44) can interact with an unknown receptor on the cell surface, to promote the mitosis of cancer cells and stimulate 
the growth of cancer cells. ${ }^{33-38}$ These studies demonstrate that CathD has an important role in controlling cell transformation and differentiation.

The in vivo data of this study showed that CathD deficiency leads to a selective loss of mature oligodendrocytes not related to apoptosis. However, as CathD has both anti-apoptotic and pro-apoptotic functions under different situations, the function of this protease in apoptosis is still paradoxical up to now..$^{30,39}$ In order to find a way to study OL development in CathD ${ }^{-/-}$ mice, primary OPC cultures were used in vitro to evacuate other factors. It was found that CathD deficiency did not inhibit oligodendrocyte maturation by promoting cell death. Both CathD ${ }^{-/-}$mice and CathD ${ }^{+/+}$mice exhibited the same number of mature oligodendrocytes in vitro, suggesting that CathD might have a regulating function in OL development.

\section{CathD and PLP transportation}

The biogenesis of the specialized myelin membrane requires intricate machinery: the glial cells are associated with the axons at the appropriate developmental time, whereas relevant mechanisms must initiate to ensure the vectorial delivery of newly synthesized myelin-membrane components to the axons. That is, a specific set of lipids and proteins have to be correctly assembled on the myelin membrane in time. ${ }^{40}$ In most cells, the majority molecules localizing to the internal vesicles of the endosomal system are destined for lysosomal degradation by lysosomal proteases. However, in OLs, LE/L compartments may be particularly useful as storage compartments, as they are able to harbor a large amount of membrane materials for myelin biogenesis in a multilamellar and multivesicularmanner. ${ }^{41}$ As a structural protein, PLP has a major role in the correct apposition of the extracellular leaflets of the membrane, thereby stabilizing the multilayered myelin membrane structure upon compaction. ${ }^{42,43}$ Other functions of PLP have been suggested as well, including a role in OL survival and adhesion relevant to migration. ${ }^{44}$ It is initially targeted to LEs/Ls and then redistributed from LEs/Ls to the plasma membrane upon receiving mature signals from neurons. ${ }^{45}$ Therefore, LEs/Ls may be required for the trafficking of PLP or other myelin contents from the membrane of distal processes to myelinate axons. LEs/Ls may also be required for OL maturation.

Our in vitro and in vivo experiments showed that more PLP accumulated in LEs/Ls but fewer PLP was distributed on the plasma membrane of OLs in the Cath ${ }^{-/-}$mice, suggesting that CathD deficiency affects the location of PLP in OLs. PLP contains a cholesterol recognition/interaction sequence and is a major cholesterol-interacting protein in oligodendrocytes. Pulse-chase experiments, together with the biochemical isolation of CHAPS-resistant membrane fractions and the use of a photoactive crosslinking cholesterol derivative, revealed that PLP was associated with myelin lipids after leaving the endoplasmic reticulum but before exiting the Golgi apparatus. These assemblies will eventually pinch off as transport vesicles from the trans-Golgi network and move to the plasma membrane, by using unknown motor proteins during vesicular transport. ${ }^{13}$ Therefore, some experiments were performed in this study to observe whether the transportation of PLP in OLs was obstructed in the CathD ${ }^{-/-}$mice. It was found that the transport of PLP from the LEs/Ls to the plasma membrane of OLs was slowed down in the CathD ${ }^{-/-}$mice, but neither the clatherin-dependent nor the clatherin-independent endocytosis of PLP was increased. However, it cannot be ruled out that CathD deficiency interferes with the transport of PLP by interfering with the transport of cholesteryl and esters.

\section{Possible interactions between CathD and PLP}

VAMP7 (also called tetanus neurotoxin-insensitive-VAMP, TIVAMP) is involved in various important cellular functions, including phagocytosis, mitosis, cell migration, autophagosome biosynthesis as well as membrane repair and growth, and it acts mainly through mediating the fusion of vesicles that are derived from Golgi, late-endosomal and lysosomal compartments with the plasma membrane. ${ }^{46-50}$ The specific role of VAMP7 in regulating the exocytosis of LEs/Ls has been well established in several cell types ${ }^{51-53}$ and appears to be primarily associated with cellular processes requiring rapid expansion and remodeling of the plasma membrane. ${ }^{54,55}$ The long $\mathrm{N}$-terminal domain (longin domain) of VAMP7 could be identified as a key factor that acts as an 'on/off switch for its activity. In PC12 cells, overexpression of the VAMP7 longin domain inhibits neurite outgrowth, whereas its deletion activates the SNARE-complex assembly and stimulates neurite outgrowth. ${ }^{56}$ In mature hippocampal neurons, removal of the longin domain increases spontaneous $\mathrm{Ca}^{2+}$-independent fusion of VAMP7-containing synaptic vesicles with the pre-synaptic plasma membrane. ${ }^{57}$ Moreover, the selective sorting of VAMP7 is mediated by direct interactions between its longin domain and the coat components of clathrin-coated vesicles. ${ }^{58}$ There are few studies on how CathD regulates PLP transport from LEs/Ls to the cell membrane in oligodendrocyte lineage cells. Recent data showed that VAMP7 colocalized with PLP in the LEs/Ls of oligodendrocyte lineage cells, and controlled the exocytosis of PLP from $\mathrm{LE} / \mathrm{L}$ organelles as a part of a transcytosis pathway, whereas the missorting of VAMP7 caused a mild dysmyelination characterized by reduced select myelin proteins, including PLP. ${ }^{17}$ In addition, VAMP7 participated in the degradative function of lysosomes by mediating proper post-Golgi maturation of CahtD. ${ }^{59}$ Thus, we hypothesized that VAMP7 may act as the mediator between CathD and PLP. Our results suggest that CathD, PLP and VAMP7 may form a binding complex, indicating that the loss of CathD may affect some interaction between CathD and longin domain of VAMP7, and lead to difficult exocytosis of PLP from LE/Ls to cell membrane of oligodendrocyte lineage cells, thus delaying the morphological maturation of oligodendrocytes. However, it remains unknown whether CathD, PLP and VAMP7 could bind directly or there are any other mechanisms. Further experiments are required to demonstrate the interactions among CathD, PLP and VAMP7.

\section{CONFLICT OF INTEREST}

The authors declare no conflict of interest. 


\section{ACKNOWLEDGEMENTS}

This study was funded by the Natural Science Foundation of China (81401063, 31471013), Beijng Nova Program (Z161100004916144), The natural Science Foundation of Beijing (7153175) and the Shanghai Pujiang Program (17PJ1410900).

\section{PUBLISHER'S NOTE}

Springer Nature remains neutral with regard to jurisdictional claims in published maps and institutional affiliations.

1 Reid WA, Valler MJ, Kay J. Immunolocalization of cathepsin D in normal and neoplastic human tissues. J Clin Pathol 1986; 39: 1323-1330.

2 Chen L, Li H, Liu W, Zhu J, Zhao X, Wright E et al. Olfactomedin 4 suppresses prostate cancer cell growth and metastasis via negative interaction with cathepsin D and SDF-1. Carcinogenesis 2011; 32: 986-994.

3 Kim SJ, Kim KH, Ahn ER, Yoo BC, Kim SY. Depletion of cathepsin D by transglutaminase 2 through protein cross-linking promotes cell survival. Amino Acids 2013; 44: 73-80.

4 Seider K, Brunke S, Schild L, Jablonowski N, Wilson D, Majer 0 et al. The facultative intracellular pathogen Candida glabrata subverts macrophage cytokine production and phagolysosome maturation. J Immunol 2011; 187: 3072-3086.

5 Fritchie K, Siintola E, Armao D, Lehesjoki AE, Marino T, Powell C et al. Novel mutation and the first prenatal screening of cathepsin D deficiency (CLN10). Acta Neuropathol 2009; 117: 201-208.

6 Trajkovic K, Dhaunchak AS, Goncalves JT, Wenzel D, Schneider A, Bunt G et al. Neuron to glia signaling triggers myelin membrane exocytosis from endosomal storage sites. J Cell Biol 2006; 172: 937-948.

7 Koike M, Nakanishi H, Saftig P, Ezaki J, Isahara K, Ohsawa Y et al. Cathepsin D deficiency induces lysosomal storage with ceroid lipofuscin in mouse CNS neurons. J Neurosci 2000; 20: 6898-6906.

8 Saftig P, Hetman M, Schmahl W, Weber K, Heine L, Mossmann $\mathrm{H}$ et al. Mice deficient for the lysosomal proteinase cathepsin $D$ exhibit progressive atrophy of the intestinal mucosa and profound destruction of lymphoid cells. EMBO J 1995; 14: 3599-3608.

9 Haapanen A, Ramadan UA, Autti T, Joensuu R, Tyynela J. In vivo MRI reveals the dynamics of pathological changes in the brains of cathepsin D-deficient mice and correlates changes in manganeseenhanced MRI with microglial activation. Magn Reson Imaging 2007; 25: 1024-1031.

10 Partanen S, Haapanen A, Kielar C, Pontikis C, Alexander N, Inkinen T et al. Synaptic changes in the thalamocortical system of cathepsin D-deficient mice: a model of human congenital neuronal ceroid-lipofuscinosis. $J$ Neuropathol Exp Neurol 2008; 67: 16-29.

11 Mutka AL, Haapanen A, Kakela R, Lindfors M, Wright AK, Inkinen T et al. Murine cathepsin $D$ deficiency is associated with dysmyelination/myelin disruption and accumulation of cholesteryl esters in the brain. $J$ Neurochem 2010; 112: 193-203.

12 Schneider A, Lander H, Schulz G, Wolburg H, Nave KA, Schulz JB et al. Palmitoylation is a sorting determinant for transport to the myelin membrane. J Cell Sci 2005; 118: 2415-2423.

13 Simons M, Kramer EM, Thiele C, Stoffel W, Trotter J. Assembly of myelin by association of proteolipid protein with cholesterol- and galactosylceramiderich membrane domains. J Cell Biol 2000; 151: 143-154.

14 Weimbs T, Stoffel W. Proteolipid protein (PLP) of CNS myelin: positions of free, disulfide-bonded, and fatty acid thioester-linked cysteine residues and implications for the membrane topology of PLP. Biochemistry 1992; 31: 12289-12296.

15 Roussel G, Neskovic NM, Trifilieff E, Artault JC, Nussbaum JL. Arrest of proteolipid transport through the Golgi apparatus in Jimpy brain. J Neurocytol 1987; 16: 195-204.

16 Snaidero N, Mobius W, Czopka T, Hekking LH, Mathisen C, Verkleij D et al. Myelin membrane wrapping of CNS axons by PI(3,4,5)P3-dependent polarized growth at the inner tongue. Cell 2014; 156: 277-290.

17 Feldmann A, Amphornrat J, Schonherr M, Winterstein C, Mobius W, Ruhwedel $\mathrm{T}$ et al. Transport of the major myelin proteolipid protein is directed by VAMP3 and VAMP7. J Neurosci 2011; 31: 5659-5672.
18 Chen Y, Balasubramaniyan V, Peng J, Hurlock EC, Tallquist M, Li J et al. Isolation and culture of rat and mouse oligodendrocyte precursor cells. Nat Protoc 2007; 2: 1044-1051.

19 Duan S, Anderson CM, Stein BA, Swanson RA. Glutamate induces rapid upregulation of astrocyte glutamate transport and cell-surface expression of GLAST. J Neurosci 1999; 19: 10193-10200.

20 Chew LJ, Coley W, Cheng Y, Gallo V. Mechanisms of regulation of oligodendrocyte development by p38 mitogen-activated protein kinase. J Neurosci 2010; 30: 11011-11027.

21 Simons M, Trotter J. Wrapping it up: the cell biology of myelination. Curr Opin Neurobiol 2007; 17: 533-540.

22 Baumann N, Pham-Dinh D. Biology of oligodendrocyte and myelin in the mammalian central nervous system. Physiol Rev 2001; 81: 871-927.

23 Masson O, Bach AS, Derocq D, Prebois C, Laurent-Matha V, Pattingre S et al. Pathophysiological functions of cathepsin D: targeting its catalytic activity versus its protein binding activity? Biochimie 2010; 92: 1635-1643.

24 Barrett AJ. Cathepsin D the lysosomal aspartic proteinase. Ciba Found Symp 1979; 37-50.

25 Siintola E, Partanen S, Stromme P, Haapanen A, Haltia M, Maehlen J et al. Cathepsin $D$ deficiency underlies congenital human neuronal ceroidlipofuscinosis. Brain 2006; 129: 1438-1445.

26 Dimou L, Gallo V. NG2-glia and their functions in the central nervous system. Glia 2015; 63: 1429-1451.

27 Zhao X, He X, Han X, Yu Y, Ye F, Chen Y et al. MicroRNA-mediated control of oligodendrocyte differentiation. Neuron 2010; 65: 612-626.

28 Zhou Q, Choi G. Anderson DJ. The bHLH transcription factor Olig2 promotes oligodendrocyte differentiation in collaboration with $\mathrm{Nkx2.2}$. Neuron 2001; 31: 791-807.

29 Franceschini I, Vitry S, Padilla F, Casanova P, Tham TN, Fukuda M et al. Migrating and myelinating potential of neural precursors engineered to overexpress PSA-NCAM. Mol Cell Neurosci 2004; 27: 151-162.

$30 \mathrm{Mi}$ S, Miller RH, Tang W, Lee X, Hu B, Wu W et al. Promotion of central nervous system remyelination by induced differentiation of oligodendrocyte precursor cells. Ann Neurol 2009; 65: 304-315.

31 McDermott JR, Gibson AM. Degradation of Alzheimer's beta-amyloid protein by human cathepsin D. Neuroreport 1996; 7: 2163-2166.

32 Grdovic N, Vidakovic M, Mihailovic M, Dinic S, Uskokovic A, Arambasic J et al. Proteolytic events in cryonecrotic cell death: proteolytic activation of endonuclease P23. Cryobiology 2010; 60: 271-280.

33 Fusek M, Vetvicka V. Mitogenic function of human procathepsin D: the role of the propeptide. Biochem J 1994; 303(Pt 3): 775-780.

34 Vashishta A, Ohri SS, Proctor M, Fusek M, Vetvicka V. Role of activation peptide of procathepsin $D$ in proliferation and invasion of lung cancer cells. Anticancer Res 2006; 26: 4163-4170.

35 Vetvicka V, Benes P, Fusek M. Procathepsin D in breast cancer: what do we know? Effects of ribozymes and other inhibitors. Cancer Gene Ther 2002; 9: 854-863.

36 Vetvicka V, Vektvickova J, Fusek M. Effect of human procathepsin D on proliferation of human cell lines. Cancer Lett 1994; 79: 131-135.

37 Vetvicka V, Vetvickova J, Benes P. Role of enzymatically inactive procathepsin D in lung cancer. Anticancer Res 2004; 24: 2739-2743.

38 Vetvicka V, Vetvickova J, Fusek M. Anti-human procathepsin D activation peptide antibodies inhibit breast cancer development. Breast Cancer Res Treat 1999; 57: 261-269.

39 Koike M, Shibata M, Ohsawa Y, Nakanishi H, Koga T, Kametaka S et al. Involvement of two different cell death pathways in retinal atrophy of cathepsin D-deficient mice. Mol Cell Neurosci 2003; 22: 146-161.

40 Szuchet S, Nielsen LL, Domowicz MS, Austin JR 2nd, Arvanitis DL. CNS myelin sheath is stochastically built by homotypic fusion of myelin membranes within the bounds of an oligodendrocyte process. J Struct Biol 2015; 190: 56-72.

41 Kippert A, Trajkovic K, Rajendran L, Ries J, Simons M. Rho regulates membrane transport in the endocytic pathway to control plasma membrane specialization in oligodendroglial cells. J Neurosci 2007; 27: 3560-3570.

42 Eng LF, Chao FC, Gerstl B, Pratt D, Tavaststjerna MG. The maturation of human white matter myelin. Fractionation of the myelin membrane proteins. Biochemistry 1968; 7: 4455-4465.

43 Norton WT, Poduslo SE. Myelination in rat brain: changes in myelin composition during brain maturation. J Neurochem 1973; 21: 759-773.

44 Edgar JM, Anderson TJ, Dickinson PJ, Barrie JA, McCulloch MC, Nave KA et al. Survival of, and competition between, oligodendrocytes expressing different alleles of the PIp gene. J Cell Biol 2002; 158: 719-729.

45 Baron W, Hoekstra D. On the biogenesis of myelin membranes: sorting, trafficking and cell polarity. FEBS Lett 2010; 584: 1760-1770. 
46 Chaineau M, Danglot L, Proux-Gillardeaux V, Galli T. Role of HRB in clathrin-dependent endocytosis. J Biol Chem 2008; 283: 34365-34373.

47 Hesketh GG, Pérez-Dorado I, Jackson LP, Wartosch L, Schäfer IB, Gray SR et al. VARP is recruited on to endosomes by direct interaction with retromer, where together they function in export to the cell surface. Dev Cell 2014; 29: 591-606.

48 Luzio JP, Parkinson MDJ, Gray SR, Bright NA. The delivery of endocytosed cargo to lysosomes. Biochem Soc Trans 2009; 37: 1019-1021.

49 Rossi V, Banfield DK, Vacca M, Dietrich LEP, Ungermann C, D’Esposito M et al. Longins and their longin domains: regulated SNAREs and multifunctional SNARE regulators. Trends Biochem Sci 2004; 29: 682-688.

50 Moreau K, Ravikumar B, Renna M, Puri C, Rubinsztein DC. Autophagosome precursor maturation requires homotypic fusion. Cell 2011; 146: 303-317.

51 Arantes RM, Andrews NW. A role for synaptotagmin VII-regulated exocytosis of lysosomes in neurite outgrowth from primary sympathetic neurons. $J$ Neurosci 2006; 26: 4630-4637.

52 Marcet-Palacios M, Odemuyiwa SO, Coughlin JJ, Garofoli D, Ewen C, Davidson CE et al. Vesicle-associated membrane protein 7 (VAMP-7) is essential for target cell killing in a natural killer cell line. Biochem Biophys Res Commun 2008; 366: 617-623.

53 Proux-Gillardeaux V, Raposo G, Irinopoulou T, Galli T. Expression of the Longin domain of TI-VAMP impairs lysosomal secretion and epithelial cell migration. Biol Cell 2007; 99: 261-271.

54 Chaineau M, Danglot L, Galli T. Multiple roles of the vesicular-SNARE TIVAMP in post-Golgi and endosomal trafficking. FEBS Lett 2009; 583: 3817-3826.

55 Danglot L, Chaineau M, Dahan M, Gendron MC, Boggetto N, Perez F et al. Role of TI-VAMP and CD82 in EGFR cell-surface dynamics and signaling. $J$ Cell Sci 2010; 123: 723-735.
56 Martinez-arca S, Alberts P, Zahraoui A, Louvard D, Galli T. Role of tetanus neurotoxin insensitive vesicle-associated membrane protein (TI-VAMP) in vesicular transport mediating neurite outgrowth. J Cell Biol 2000; 149: 889-899.

57 Hua Z, Leal-Ortiz S, Foss SM, Waites CL, Garner CC, Voglmaier SM et al. v-SNARE composition distinguishes synaptic vesicle pools. Neuron 2011 . 71: 474-487.

58 Martinez-Arca S, Rudge R, Vacca M, Raposo G, Camonis J, ProuxGillardeaux $\mathrm{V}$ et al. A dual mechanism controlling the localization and function of exocyticv-SNAREs. Proc Natl Acad Sci USA 2003; 100 : 9011-9016.

59 Okumura AJ, Hatsuzawa K, Tamura T, Nagaya H, Saeki K, Okumura F et al. Involvement of a novel Q-SNARE, D12, in quality control of the endomembrane system. J Biol Chem 2006; 281(7): 4495-4506.

(c) (1) () $\Theta$ This work is licensed under a Creative Commons Attribution-NonCommercial-NoDerivs 4.0 International License. The images or other third party material in this article are included in the article's Creative Commons license, unless indicated otherwise in the credit line; if the material is not included under the Creative Commons license, users will need to obtain permission from the license holder to reproduce the material. To view a copy of this license, visit http://creativecommons.org/licenses/by-nc-nd/4.0/

(C) The Author(s) 2018 